\title{
PK/PD-based adaptive tailoring of oseltamivir doses to treat within-host influenza viral infections
}

\author{
Ghazal Montaseria ${ }^{\mathrm{a}, \mathrm{b}}$, Alessandro Boianelli ${ }^{\mathrm{c}, \mathrm{a}}$, Esteban A. Hernandez-Vargas ${ }^{\mathrm{d}, \mathrm{a}, *}$, \\ Michael Meyer-Hermann ${ }^{\mathrm{a}, \mathrm{e}, \mathrm{b}, *}$ \\ ${ }^{a}$ Department of Systems Immunology and Braunschweig Integrated Centre of Systems Biology, \\ Helmholtz Centre for Infection Research, Braunschweig, Germany \\ ${ }^{b}$ Centre for Individualised Infection Medicine (CIIM), Hannover, Germany \\ ${ }^{c}$ Department of Drug Discovery Sciences, Boehringer Ingelheim Pharma GmbH \&5 Co. KG, \\ Biberach an der Riß, Germany \\ ${ }^{d}$ Frankfurt Institute for Advanced Studies, Frankfurt am Main, Germany \\ ${ }^{e}$ Institute for Biochemistry, Biotechnology and Bioinformatics, Technische Universität \\ Braunschweig, Braunschweig, Germany
}

\begin{abstract}
Influenza A virus (IAV) is a latent global threat to human health. In view of the risk of pandemics, prophylactic and curative treatments are essential. Oseltamivir is a neuraminidase inhibitor efficiently supporting recovery from influenza infections. Current common clinical practice is a constant drug dose (75 or $150 \mathrm{mg}$ ) administered at regular time intervals twice a day. We aim to use quantitative systems pharmacology to propose an efficient adaptive drug scheduling. We combined the mathematical model for IAV infections validated by murine data, which captures the viral dynamics and the dynamics of the immune host response, with a pharmacokinetic (PK)/pharmacodynamic (PD) model of oseltamivir. Next, we applied an adaptive impulsive feedback control method to systematically calculate the adaptive dose of oseltamivir in dependence on the viral load and the number of immune effectors at the time of drug administration. Our in silico results revealed that the treatment with adaptive control-based drug scheduling is able to either increase the drug virological efficacy or reduce the drug dose while keeping the same virological efficacy. Thus, adaptive adjustment of the drug dose would reduce not only the potential side effects but also the amount of stored oseltamivir required for the prevention of outbreaks.
\end{abstract}

Keywords: Impulsive control; Influenza viral infections; PK/PD modeling; Oseltamivir

\footnotetext{
* Corresponding authors.

Email addresses: ghazal.montaseri@helmholtz-hzi.de (Ghazal Montaseri), alessandro.boianelli@boehringer-ingelheim.com (Alessandro Boianelli), vargas@fias.uni-frankfurt.de (Esteban A. Hernandez-Vargas), mmh@theoretical-biology.de (Michael Meyer-Hermann)
} 


\section{Introduction}

The pandemic outbreaks of Influenza A virus (IAV) revealed the lethality of the virus causing high morbidity and mortality worldwide (World Health Organization, 2009; Potter, 2001; Kilbourne, 2006). Influenza vaccination (Centers for Disease Control and Prevention, 2018), the most effective intervention so far, showed inadequate effectiveness against the last pandemic IAV strain H1N1. A matching vaccine for a particular IAV strain is often not available in the short term. Beside influenza vaccination, antiviral therapy with neuraminidase inhibitors remains the most effective strategy against IAV (Gubareva et al., 2000). The neuraminidase inhibitor most frequently stockpiled worldwide for the purpose of preparation on IAV pandemic outbreaks and protection of the world's population is oseltamivir. After absorption in the host, the pro-drug oseltamivir phosphate is converted into the active metabolite oseltamivir carboxylate which blocks the viral release from infected cells (Wattanagoon et al., 2009; Boianelli et al., 2016). Oseltamivir reduces symptoms and secondary IAV transmission.

Antiviral scheduling may be an important approach to combat influenza infections. Current clinical practice is a constant drug dose administered at regular time intervals of twice a day. While the drug inhibits the spread of the virus in the organism, it cooperates with the immune system to control the viral load and virus clearance. The interactions between the virus, the immune response, and the drug are classical complex systems which can be investigated by methods from systems immunology and control engineering. The potential of control engineering approaches to improve disease treatment has been shown for diabetes (Dhumpa et al., 2014; Doyle et al., 2014; Cobelli et al., 2011; Teixeira and Malin, 2008; Jacobs et al., 2011), cancer (Swan, 1990; Martin and Teo, 1993; Martin, 1992; Itik et al., 2009), and HIV (Chang and Astolfi, 2008; Zurakowski and Teel, 2006; Cardozo and Zurakowski, 2012; Hernandez-Vargas et al., 2014a, 2013). It is the aim of this work to tailor the required dose of oseltamivir based on adaptive feedback control of the administered drug dose.

Optimization of drug regimens requires a systems immunology approach that includes the pharmacokinetics (PKs) of drug delivery and absorption, the pharmacodynamics (PDs) of drug activity against the pathogen in the organism, the replication of the virus $(\mathrm{V})$ and the action of the immune effector cells (E). PK/PD models have been employed at all stages of drug discovery and application by pharmaceutical companies (van der Graaf and Benson, 2011; Schaller et al., 2016) in order to improve drug efficacy and productivity. The dynamics of the viral infection and the immune response are handled by sets of differential equations and have been developed for IAV (Handel et al., 2007; Hancioglu et al., 2007; Saenz et al., 2010; Lee et al., 2009; Miao et al., 2010; Baccam et al., 2006; Dobrovolny et al., 2013; Hernandez-Vargas et al., 2014b; Pawelek et al., 2012; Price et al., 2015). These models highlight the role of NK cells for controlling the virus and of CD8+ T cells (CTL) to clear IAV (Miao et al., 2010; Hernandez-Vargas et al., 2014b). However, only a few modeling studies have investigated quantitatively the efficacy of different treatment strategies based on oseltamivir (Canini et al., 2014; Holder et al., 2011; Dobrovolny et al., 
Figure 1: Scheme of adaptive feedback control loop. The closed-loop interaction between the drug, the virus and the immune response is shown. Gray dashed box: The virus replicates at rate $p$ (logistic growth, see Subsection 2.1) and induces more immune effector cells (collected here as CTLs) at rate $r$. CTLs suppress viral replication at rate $c_{v}$, die at rate $c_{e}$, and are replenished at rate $S_{E}$. The drug inhibits viral replication at rate $p$. The appropriate dose $u$ is calculated adaptively in the control-based drug dose unit from the measured virus concentration and the number of CTLs at each time of drug administration.

2011).

If we cast the problem of drug dose design in a control engineering framework, we may benefit from feedback control methods such as proportional-integralderivative (PID) control (Ogata, 2010), feedback linearization method (Isidori, 1995) (used along with the impulsive control method (Yang, 2001; Rivadeneira and Moog, 2012) in this study), sliding mode control (Isidori, 1995), passivitybased control design (Khalil, 2002), singular perturbation method (Kokotović et al., 1999) and backstepping techniques (Zhou and Wen, 2008). Depending on the structure of the disease model, specific feedback control methods are applicable to calculate the drug dose. For instance, these techniques have been largely used for the treatment scheduling of HIV infection modeled by various mathematical models (Chang and Astolfi, 2008; Zurakowski and Teel, 2006; Cardozo and Zurakowski, 2012; Hernandez-Vargas et al., 2014a, 2013). However, to the best of the authors knowledge, there is no work discussing control-based methods to treat influenza viral infections.

The above-mentioned control-based works on the treatment scheduling of HIV infection have shed light on how to improve current antiretroviral guidelines to mitigate virus mutation. In this study, control-based oseltamivir scheduling for treating influenza infection was considered. Technically, the feedback linearization method (Isidori, 1995; Khalil, 2002) was applied in the framework of impulsive control systems (Rivadeneira and Moog, 2012) to the virus-immune model (Boianelli et al., 2015). The resulting linear model is stabilized with the pole placement method (Yang, 2001) (see also Appendix A).

Thus far, optimal control-based methods designed for influenza therapy have been proposed between host levels (epidemiology) (Alexander et al., 2007; Lee et al., 2010; Tchuenche et al., 2011; Gubar and Zhu, 2013; Lee et al., 2013; Jaberi-Douraki and Moghadas, 2014). Here, we investigate within-host drug scheduling using the IAV-immune model in (Boianelli et al., 2015). In this paper, we combined the mathematical model for IAV infections (Boianelli et al., 2015) validated by murine data (Toapanta and Ross, 2009), that captures the viral dynamics and those of the immune effector response (Fig. 1, gray dashed box), with a PK/PD model. Next, we applied an adaptive feedback control method to calculate the optimal dose of oseltamivir depending on the viral load and the number of immune effectors at the time of drug administration (Fig. 1). The results contribute to develop an adaptive treatment of IAV infections in a clinical context, which requires further investigations. 
Table 1: Model parameters.

\begin{tabular}{llll}
\hline Parameter & Nominal value & Units & Reference \\
\hline$r$ & 0.33 & days $^{-1}$ & (Boianelli et al., 2015) \\
$k_{e}$ & $2.7 \times 10^{3}$ & PFU $/ \mathrm{ml}$ & (Boianelli et al., 2015) \\
$c_{e}$ & $2 \times 10^{-2}$ & days $^{-1}$ & (McDonagh and Bell, 1995) \\
$S_{E}$ & $2 \times 10^{4}$ & days $^{-1}$ cells & (Boianelli et al., 2015) \\
$p$ & 4.4 & day $^{-1}$ & (Boianelli et al., 2015) \\
$k_{v}$ & $10^{6}$ & $\mathrm{PFU} / \mathrm{ml}^{-1}$ & (Toapanta and Ross, 2009) \\
$c_{v}$ & $1.24 \times 10^{-6}$ & days $^{-1}$ cells & (Boianelli et al., 2015) \\
$E(0)$ & $10^{6}$ & cells $^{-1}$ & (Boianelli et al., 2015) \\
$V(0)$ & 25 & PFU $/ \mathrm{ml}$ & (Boianelli et al., 2015) \\
\hline
\end{tabular}

\section{Material and methods}

\subsection{Mathematical model of IAV dynamics}

The model of viral and immune dynamics in response to IAV infections was shown to have identifiable parameters (Boianelli et al., 2015) based on the murine data (Toapanta and Ross, 2009). It reads (Fig. 1, gray dashed box)

$$
\begin{aligned}
& \dot{E}=r E\left(\frac{V}{V+k_{e}}\right)-c_{e} E+S_{E} \\
& \dot{V}=p V\left(1-\frac{V}{k_{v}}\right)-c_{v} V E
\end{aligned}
$$

where $E$ and $V$ represent CD8+ T cells (CTLs) and IAV, respectively. The homeostatic replenishment of CTLs is given by $S_{E}=c_{e} E(0)$, where $E(0)$ is the steady-state number of CTLs in the absence of virus and $c_{e}$ is their halflife. The virus-dependent proliferation of CTLs is represented by a Michaelis Menten function with a constant half saturation parameter $k_{e}$. The Hill function models the dependency of the immune cells recruitment on the infectious dose as investigated in (Marois et al., 2012), and the Hill coefficient $n$ adjusts the steepness of the immune response to the infectious dose. As reported in (Boianelli et al., 2015) and the following simulation results, the adaptive immune system responds to the virus with one day delay. This implies that an extra delay of the onset of the immune response is not needed in the model to make it consistent with the experimental data.

The virus $V$ replicates according to a logistic function with a maximum carrying capacity $k_{v}$ and the growth rate $p$. Influenza virus is cleared at a rate $c_{v} E$. In general, the term $-c_{v} V E$ may indicate an infinite killing capacity of CTLs. However, the experimental data used in (Boianelli et al., 2015) is in a regime where CTLs do not exceed their maximum capacity for viral clearance. If this is not the case for other data, the virus killing capacity of CTLs should be limited by a saturation function of the viral load. Parameter values are listed in Table 1. Without any treatment, the immune system is able to clear the 
Table 2: Oseltamivir PK/PD parameters.

\begin{tabular}{llll}
\hline Parameter & Nominal value & Units & Reference \\
\hline$D_{50}$ & 42.30 & $\mathrm{mg}$ & (Tamiflu, 2009) \\
$\gamma$ & 3.26 & days $^{-1}$ & (Wattanagoon et al., 2009) \\
$\tau_{k+1}-\tau_{k}$ & 0.5 & days & (World Health Organization, 2009) \\
\hline
\end{tabular}

virus. However, treatment can enhance the performance of the immune system in terms of higher virological efficacy.

\subsection{Oseltamivir $P K / P D$ dynamics}

Drug administration involves PK and PD (Mhawej et al., 2010; Legrand et al., 2003). PKs are modeled by $\dot{D}=-\gamma D$ (between any two consecutive doses) and describes a complete and instantaneous absorption of the drug by first-order kinetics. $D$ is the amount of the drug in the blood and $\gamma$ is its elimination rate. PDs are modeled as $\epsilon=\frac{D}{D+D_{50}}$, which reflects the efficacy of the treatment. The parameter $D_{50}$ is the drug concentration at which $\epsilon=0.5 . \tau_{k}, k=1,2, \ldots$ is the time sequence at which the drug is taken. Since oseltamivir inhibits viral expansion, we modify the viral replication rate $p$ to $p(1-\epsilon)$. Thus, (1) becomes

$$
\dot{V}=p(1-\epsilon) V\left(1-\frac{V}{k_{v}}\right)-c_{v} V E
$$

where the drug dynamics are described by

$$
\dot{D}=-\gamma D, \quad \tau_{k} \leqslant t<\tau_{k+1}, k=1,2, \ldots
$$

between any two intake times. The PK/PD parameter values are listed in Table 2. Note that the absorption rate of oseltamivir (about 1 hour) is fast compared to its elimination rate (7.35 hours) (Wattanagoon et al., 2009), justifying (3).

Let $\tau_{k}^{-}$and $\tau_{k}^{+}$refer to time instants before and after taking the drug at time $\tau_{k}$, respectively. Then, each interval starting at $\tau_{k}$ is associated with a drug dose $\Delta \mathrm{D}\left(\tau_{\mathrm{k}}\right)$, which is given by

$$
\Delta \mathrm{D}\left(\tau_{k}\right)=D\left(\tau_{k}^{+}\right)-D\left(\tau_{k}^{-}\right)=u\left(\tau_{k}\right), k=1,2, \ldots
$$

where $D\left(\tau_{k}^{+}\right)$is the drug concentration in the body after taking oseltamivir and $D\left(\tau_{k}^{-}\right)$is the remaining drug in the body at the intake time $\tau_{k}$. We assume, $D\left(\tau_{1}^{-}\right)=0$ (no drug is in the body before taking the first dose). Note that after taking the first dose, considering the half-life of oseltamivir absorption and results of (He et al., 1999; Morrison et al., 2007), the concentration of the drug just before the next dose is not zero, i.e., $D\left(\tau_{k}^{-}\right) \neq 0, k=2,3, \ldots$. The administered adaptive dose $u\left(\tau_{k}\right)$ is calculated at each time point from the viral load and the number of immune effector cells (see Appendix A.2). The drug administration frequency is twice a day, unless otherwise stated. 


\subsection{Treatment strategies}

The World Health Organization (WHO) recommends two treatment regimens for oseltamivir (World Health Organization, 2009) with $\Delta \mathrm{D}_{\text {fixed }}=75 \mathrm{mg}$ or, for pandemic strains, two doses per day with $\Delta \mathrm{D}_{\text {fixed }}=150 \mathrm{mg}$ per dose. $\Delta \mathrm{D}=150$ $\mathrm{mg}$ is the approved drug dose without toxicity (Wattanagoon et al., 2009) and can be considered as a safe oseltamivir dose. In the following, these two treatments will be denoted as low and high dose common clinical practice and will serve as a reference for the control-based treatment derived from adaptive feedback control method. Note that the adaptively calculated dose may be larger than the doses recommended by WHO. In order to account for the safe upper dose limit and for better comparison with the common clinical practice, in a second scenario, the applied drug is limited to the WHO recommended dose $\left(\Delta \mathrm{D}_{\text {adaptive }} \leq 75\right.$ or $150 \mathrm{mg}$ ), denoted as safe control-based treatment.

\subsection{Quantification of treatment success}

To compare the different treatment strategies, we define two read-outs. The virological efficacy index $\Gamma$ reflects the improvement in the viral load by the treatment. It was defined in (Canini et al., 2014) as

$$
\Gamma=100\left(1-\frac{S_{+}}{S_{-}}\right) \%,
$$

where $S_{+}$and $S_{-}$are the area under the $V$ curve (not in the $\log _{10}$ scale) with and without treatment, respectively, and derived from time zero to the time at which the viral load becomes less than 50 copies per ml.

The second index $\mathrm{S}_{\mathrm{D}}$ is defined as the area under the drug $D$ curve and reflects the total amount of administered drugs. It is computed from the time of first drug administration to the end of treatment. The treatment is continued for a period of 7 days from the first dose.

\subsection{Population approach}

To check the robustness of different treatment scenarios, we performed Monte Carlo analysis (Canini et al., 2014). We selected all parameters in the IAVimmune model with treatment uniformly within the interval $[\bar{m}-20 \% \bar{m}, \bar{m}+20 \% \bar{m}]$; where $\bar{m}$ is the median value of $m$ with $m \in\left\{r, k_{e}, c_{e}, p, k_{v}, c_{v}, D_{50}, \gamma\right\}$. In addition, initial states $V(0)$ and $E(0)$ are selected uniformly based on the previous constraint. Note that since $E$ is the number of cells, we round the generated number from the random process toward the nearest integer. To express the drug parameters $\gamma$ and $D_{50}$ in appropriate units in agreement with other units, some unit conversions are performed on the original values provided in (Wattanagoon et al., 2009) and (Tamiflu, 2009). The unit of $\gamma$ is converted from hours to days. $D_{50}$ is in the range of $0.0008 \mu \mathrm{M}$ to $35 \mu \mathrm{M}$ (Tamiflu, 2009). It is converted from $\mu \mathrm{M}$ to mg using $1 \mu \mathrm{M}=0.284 \mu \mathrm{g} / \mathrm{mL}$, volume of distribution $=3.31 \mathrm{~L} / \mathrm{Kg}$ (Wattanagoon et al., 2009) and the standard body weight of $75 \mathrm{Kg}$. 
Figure 2: Low dose oseltamivir treatments started on day 3 after infection. a) Immune response, b) viral load and c) drug dynamics under the three different drug administration strategies for the low dose common clinical practice with $\Delta \mathrm{D}_{\text {fixed }}=75 \mathrm{mg}$ and imposing $\Delta \mathrm{D}_{\text {adaptive }} \leq 75$ $\mathrm{mg}$ in the safe control-based treatment. The viral infection is assumed to be cleared when $V \leq 50$ copies per $\mathrm{ml}$ are reached. Note that in the long term, the CTL count in the model returns to healthy conditions (not shown). Compared to the common clinical practice, the control-based treatment clears the infection equally fast, while keeping the number of CTLs lower. Under the safe control-based treatment, viral clearance is delayed by one day, but the drug dose is visibly reduced at later times of the infection.

\subsection{Statistical analysis}

We performed a one-way ANOVA test (Rice, 2007) to compare the statistical significance of the virological efficacy index $\Gamma$ and the area under the drug curve $\mathrm{S}_{\mathrm{D}}$ between the three mentioned treatment strategies.

\section{Results}

First, we considered the low dose common clinical practice starting treatment on day 3 after infection when the symptoms of influenza infection are prominent. The common clinical practice (Fig. 2, green dashed curves) clears the viral infection earlier than without treatment (Fig. 2, black bold solid curves) while reducing the strength of the immune response.

In comparison, the control-based treatment (Fig. 2, blue dotted curves) clears the infection equally fast while keeping the number of effector cells even lower than the common clinical practice. Quantification of the virological efficacy and the administered drug revealed that the control-based treatment used about the same drug amount as in the common clinical practice but with a higher virological efficacy of $79 \%$ instead of $71 \%$ (Table 3). When the total drug taken in the control-based treatment is distributed into equal doses, i.e. $83 \mathrm{mg}$ per dose, the virological efficacy is not restored but increases only to $72 \%$ (the third row of Table 3), further underpinning the relevance of the adaptation.

However, the peak doses calculated from adaptive control theory were above the safety limit of $150 \mathrm{mg}$ (Fig. 2c, blue dotted curve). When limiting the

Table 3: Low dose oseltamivir treatments started on day 3 after infection. Comparison of the virological efficacy index $\Gamma$ and total administered drug (area under the drug curve $\mathrm{S}_{\mathrm{D}}$ ) for the different treatments. We assume $\Delta \mathrm{D}_{\text {adaptive }} \leq \Delta \mathrm{D}_{\text {fixed }}=75 \mathrm{mg}$. Data are derived partially from Fig. 2.

\begin{tabular}{lll}
\hline Treatment strategies & $\Gamma$ & $\mathrm{S}_{\mathrm{D}}$ \\
\hline Common clinical practice & $71 \%$ & 316 \\
Control-based treatment & $79 \%$ & 349 \\
Fixed dose treatment with $83 \mathrm{mg}$ & $72 \%$ & 349 \\
Safe control-based treatment & $71 \%$ & 155 \\
Fixed dose treatment with $37 \mathrm{mg}$ & $56 \%$ & 155 \\
\hline
\end{tabular}


Figure 3: High dose oseltamivir treatments started on day 3 after infection. a) Immune response, b) viral load and c) drug dynamics under the three different drug administration strategies for the high dose common clinical practice with $\Delta \mathrm{D}_{\text {fixed }}=150 \mathrm{mg}$ and imposing $\Delta \mathrm{D}_{\text {adaptive }} \leq 150$ $\mathrm{mg}$ in the safe control-based treatment. The overall higher dose reduces differences in the CTLs level in all cases. The safe control-based treatment clears the infection equally fast compared to the other two treatment strategies with a lower amount of the drug.

adaptive dose to $\Delta \mathrm{D}_{\text {adaptive }} \leq 75 \mathrm{mg}$, viral clearance was delayed by one day (Fig. 2b, red thin solid curve), but with visibly reduced drug doses at later times of the infection (Fig. 2c, red solid curve). After day 6 of the infection, the adaptive drug amount drops to $11 \mathrm{mg}$ per dose. Quantification of these results showed that the virological efficacy is back to the level of the common clinical practice but using only half of the drugs (Table 3). Conversely, if the total drug taken in the safe control-based treatment was distributed into equal doses over the treatment period, i.e. $37 \mathrm{mg}$ per dose, the virological efficacy would drop to $56 \%$ (the last row of Table 3 ) demonstrating the potential of the adaptive dose control.

Next, we repeated the same analysis starting from the high dose common clinical practice, thus using $150 \mathrm{mg}$ per dose (Fig. 3). The overall higher dose reduces differences in the immune response between the different treatments. Viral clearance is achieved at similar times in all cases. The quantification of virological efficacy and the total amount of administered drugs (Table 4) revealed that the overall virological efficacy is increased from $79 \%$ with the common clinical practice to $82 \%$ with the control-based treatment. Otherwise, the results at $75 \mathrm{mg}$ per dose were confirmed. In comparison to the common clinical practice, the control-based treatment increases virological efficacy to $82 \%$ with the same amount of total drug, and the safe control-based treatment keeps the same virological efficacy of $79 \%$ with the administration of only $2 / 3$ of oseltamivir in total. For a direct comparison of low and and high dose results refer to Supplementary Fig. S1.

The incubation period of the virus varies between 1-4 days and infected hosts may be symptomatic at different days after the establishment of infection (Centers for Disease Control and Prevention, 2016). Thus, the time of treatment initiation may be crucial for drug efficacy. We investigated all three treatment

Table 4: High dose oseltamivir treatments started on day 3 after infection. Comparison of the virological efficacy index $\Gamma$ and total administered drug (area under the drug curve $S_{D}$ ) for the three different treatments. We assume $\Delta \mathrm{D}_{\text {adaptive }} \leq \Delta \mathrm{D}_{\text {fixed }}=150 \mathrm{mg}$. Data are derived from Fig. 3.

\begin{tabular}{lll}
\hline Treatment strategies & $\Gamma$ & $\mathrm{S}_{\mathrm{D}}$ \\
\hline Common clinical practice & $79 \%$ & 633 \\
Control-based treatment & $82 \%$ & 631 \\
Safe control-based treatment & $79 \%$ & 422 \\
\hline
\end{tabular}


Table 5: Low dose oseltamivir treatments started on day 2 or 4 after infection. Comparison of the virological efficacy index $\Gamma$ and total administered drug (area under the drug curve $\mathrm{S}_{\mathrm{D}}$ ) for the three different treatments. We assume $\Delta \mathrm{D}_{\text {adaptive }} \leq \Delta \mathrm{D}_{\text {fixed }}=75 \mathrm{mg}$. Data are derived from Supplementary figures S2 and S3.

\begin{tabular}{lllll}
\hline Starting day of treatments & \multicolumn{2}{c}{ day 2} & \multicolumn{2}{c}{ day 4} \\
\hline & $\Gamma$ & $\mathrm{S}_{\mathrm{D}}$ & $\Gamma$ & $\mathrm{S}_{\mathrm{D}}$ \\
\hline Common clinical practice & $93 \%$ & 316 & $38 \%$ & 316 \\
Control-based treatment & $97 \%$ & 555 & $43 \%$ & 271 \\
Safe control-based treatment & $93 \%$ & 224 & $38 \%$ & 139 \\
\hline
\end{tabular}

strategies under low dose condition starting on day 2 and 4 after infection (Supplementary Fig. S2 and Fig. S3). An earlier diagnosis independent of the selected treatment strategy hits the organism in a state of low virus-induced CTL activity with low CTL numbers (Supplementary Fig. S2a). This has implications for the onset of the control-based treatment, which adaptively chooses higher amounts of the drug in order to compensate for the weak immune response (Supplementary Fig. S2c). The higher amounts of the drug persist to the end of the treatment. Thus, the drug has taken over the clearance of the infection. The associated CTL counts are rather low (Supplementary Fig. S2a, blue dotted curve). This happens because the early application of oseltamivir keeps the viral load low throughout the infection, thus preventing the proper mounting of an immune response. It is not surprising that this higher amount of administered oseltamivir is associated with a high virological efficacy of $97 \%$ (Table 5). The safe control-based treatment achieves $93 \%$ with substantially lower drug amounts.

Starting the treatment on day 4 post infection reduces the overall efficacy of oseltamivir to levels around $40 \%$. As the control-based treatment adapts to the higher CTL counts at this time, this strategy achieves a higher virological efficacy of $43 \%$ compared to $38 \%$ with a lower amount of administered drugs (Table 5). The adaptive strategy now uses the active immune response and limits the addition of oseltamivir to a minimum. At late time points, the safe control-based treatment suggests doses around $8 \mathrm{mg}$ less compared to day 3 .

Next, we asked whether the advantage of the safe control-based treatment to achieve the same virological efficacy using lower total drug amounts would persist when starting the treatment either on day 2 or 4 after infection. At both time points, the safe control-based treatment achieves exactly the level of common clinical practice but with less oseltamivir $(2 / 3$ and $2 / 5$ starting on day 2 or 4 , respectively, see Table 5$)$. This clearly demonstrates that the advantage in the total amount of required oseltamivir is a robust result.

Interestingly, when treatment is initiated on day 2, the virological efficacy of both control-based treatment and safe control-based treatment present median values which are almost two folds higher than those of the in silico results in (Canini et al., 2014), where the authors evaluated the impact of oseltamivir treatment strategies in presence of a resistant strain emergence. Moreover, 
Figure 4: Monte Carlo simulations (with uniform distribution) of low dose treatments. Histograms of virological efficacy index $\Gamma$ and total administered drug (area under the drug curve $\mathrm{S}_{\mathrm{D}}$ ) of Monte Carlo analysis reflecting the diversity of infected hosts. The different treatment strategies are shown with the same color code and line style as before. Treatment was started on day 2,3 , or 4 in panels $(\mathrm{a}, \mathrm{b}),(\mathrm{c}, \mathrm{d})$, and $(\mathrm{e}, \mathrm{f})$, respectively. Simulations were performed with $\Delta \mathrm{D}_{\text {fixed }}=75 \mathrm{mg}$ for the low dose common clinical practice and with $\Delta \mathrm{D}_{\text {adaptive }} \leq 75 \mathrm{mg}$ for the safe control-based treatment. $(*)$ denotes the statistical significance between different treatments based on the one-way ANOVA test $\left(\mathrm{p}\right.$-value $\left.<\frac{0.05}{3}\right)$. The control-based treatment in a,c,e and the safe control-based treatment in b,d,f show better performances compared to the common clinical practice.

when therapy is initiated on day 4 with $75 \mathrm{mg}$, our control based treatments offer a virological efficacy value of $40 \%$ compared to $3.6 \%$ in (Canini et al., 2014). The mathematical model proposed by Canini et al. was fitted to different experimental data than those fitted in (Boianelli et al., 2015). In addition, the model in (Canini et al., 2014) had different underlying assumptions: they modeled the components of the innate immune system, like pro-inflammatory cytokines and NK cells instead of CTLs addressed here; and they considered oseltamivir-resistant viruses in addition to the normal virus. The most important difference is that here, we show the potential of adaptive drug delivery while Canini et al. used constant drug doses.

Comparing the results of different treatment initiation times underlines that the starting day of treatment does not significantly affect the viral load clearance time. Here, we continued all the treatments until 7 days after diagnosis to ensure viral load clearance. However, this treatment duration might not be optimal. The current algorithm to calculate the drug dose can neither minimize the viral load clearance time nor predict the optimal length of treatment.

Another source of uncertainty of our results might stem from the particular choice of model parameters, or equally from the diversity of parameters associated with individual infected hosts. This was analyzed by performing 10000 Monte Carlo simulations with parameters varying in a range of $\pm 20 \%$ of the values given in Table $1, \gamma$ and $D_{50}$ (see Supplementary Fig. S4). The Monte Carlo simulations generate distributions of virological efficacy and the total amount of administered oseltamivir started at different time points (Fig. 4 and Supplementary Fig. S5 for low dose and high dose treatments, respectively). We found that an earlier diagnosis improves the overall virological efficacy (Fig. 4a,c,e). The earlier diagnosis also increases the total amount of administered drug (Fig. 4b,d,f). The control-based treatment leads to a significantly higher level of virological efficacy. In addition, adaptive strategies encounter a lower immune activity and compensate for this deficiency by higher adaptive doses.

The control-based treatment exhibits a wider range of total drug amounts than the other two strategies. This result reflects that the total drug is dominated by the given dose $\Delta \mathrm{D}_{\text {fixed }}$. Even for the safe control-based treatment, the dose is bounded by the condition $\Delta \mathrm{D}_{\text {adaptive }} \leq \Delta \mathrm{D}_{\text {fixed }}$, thus reducing the variability in the total dose. Note that the total amount of administered oseltamivir is significantly lower in the safe control-based treatment than in the 
common clinical practice (Fig. 4b,d,f, red solid lines and green dashed lines). Thus, the conclusion that a significant reduction of the total amount of used oseltamivir is possible without affecting virological efficacy is confirmed in this setting that reflects the patient diversity. This is also found based on high dose treatments (Supplementary Fig. S5b,d,f, red solid lines and green dashed lines). The quantitative values of the virological efficacy and the total administered oseltamivir are summarized in Supplementary Table S1.

In the aforementioned Monte Carlo simulations, we assumed that the interpatient variability follows a uniform distribution of parameters. If the parameters are sampled from a normal distribution (Supplementary Fig. S6), the previous observations and trends in the efficiency of different treatment strategies are confirmed (Supplementary figures S7 and S8). For the normal distribution, values of $\Gamma$ are slightly higher compared to the corresponding values for the uniform distribution. This is due to the fact that parameters sampled from a normal distribution are close to the median for which the optimal results were obtained. As a consequence, values of $\gamma$ and $\mathrm{S}_{\mathrm{D}}$ obtained in the Monte Carlo simulations (with normal distribution, see Supplementary Table S2) resemble the corresponding values obtained based on the median values of parameters (Tables 3-5).

So far, we assumed that the drug is administered at regular time intervals twice a day (similar to those proposed in the common clinical practice.) At each time of drug administration, we calculate (update) the drug dose using the available measurements. To investigate the robustness of the results against drug updating intervals, we increase the time intervals of the drug dose update from twice a day to once a day, every 1.5 days and every 2 days (Fig. 5). Once the drug dose is updated, the updated dose will be administered every 12 hours until the next time of update. Using Monte Carlo simulations, Fig. 5a shows that different time intervals of the drug dose update do not affect the virological efficacy index $\Gamma$. Independent of the drug dose updating frequency, the control-based treatment has the highest $\Gamma$. If we consider the results of updating twice a day as optimal (i.e., the first three bars in Fig. 5b), then reducing the number of updates leads to a higher total administered drug $\mathrm{S}_{\mathrm{D}}$ under the control-based treatment and the safe control-based treatment. Thus, although the results are not optimal anymore, they still preserve their good performances compared to the common clinical practice.

\section{Discussion}

The optimization of treatment protocols with control engineering methodologies is not without precedent. What has proven successful in the context of chronic infections like HIV may well be useful for acute infections with IAV. Here, we provided evidence that with adaptive control-based drug scheduling the same virological efficacy can be achieved with about half of the total drug. The total amount of oseltamivir needed for treatment is an important quantity not only for the planning of pandemic outbreaks but also for minimization of potential side effects in the patient. Also, the economic burden of oseltamivir 
Figure 5: Low dose oseltamivir treatments (started on day 3 after infection) with different time intervals of drug dose update. Histograms and error bars of a) virological efficacy index $\Gamma$ and $b$ ) total administered drug (area under the drug curve $\mathrm{S}_{\mathrm{D}}$ ) for Monte Carlo simulations under the three different drug administration strategies for the low dose common clinical practice with $\Delta \mathrm{D}_{\text {fixed }}=75 \mathrm{mg}$ and imposing $\Delta \mathrm{D}_{\text {adaptive }} \leq 75 \mathrm{mg}$ in the safe control-based treatment. $(*)$ denotes the statistical significance between different treatments (with the same time interval of the drug update) based on the one-way ANOVA test (p-value $<\frac{0.05}{3}$ ). The adaptive strategies are robust against drug updating time intervals. The control-based treatment and the safe control-based treatment show respectively higher and nearly equal $\Gamma$ compared to the common clinical practice for all updating intervals. Even though they choose higher doses as the updating interval increases, their good performances are preserved.

stock-piling might be considered as an issue. However, the economic savings by reduced total drug amounts are currently compensated by increased costs in diagnosis.

Adaptive drug dose scheduling is best achieved by repeated measurements of the viral load and immune effector counts. The viral load can be determined from nasal or throat swab specimens or nasopharyngeal aspirate (with typically higher yield) and subsequent quantification by a variety of methods based on: 1) polymerase chain reaction (PCR); i.e., reverse transcriptase-PCR (RT-PCR), real-time PCR, multiplex PCR; 2) nucleic acid sequence-based methods, i.e., DNA microarray, pyrosequencing, nucleic acid sequencing or loop-mediated isothermal amplification methods (Wang and Taubenberger, 2010); or 3) direct fluorescence antibody assay (DFA) (Kim and Poudel, 2013; Peaper and Landry, 2014). While PCR-based methods require amplification of viral DNA, DFA involves the staining of cells from the sample followed by bioconjugation of antibodies to the fluorescent dye. In comparison to PCR-based assays, DFA confers cheaper results, although it is not as sensitive as the PCR test (Kim and Poudel, 2013). Thus, real-time RT-PCR is presently the most sensitive detection technique for fast, specific and high-throughput viral load quantification. While the viral load can be quantitatively calculated in around 60 minutes with realtime PCR, for the purpose discussed in the present manuscript, a rapid molecular assay that can be performed in 20 minutes would be more appropriate (Binnicker et al., 2015).

The state of the immune response might be derived from blood samples using a complete blood count. Note that immune effector counts are not necessarily restricted to CTL counts, but may be replaced by measurements of any correlated quantity. White blood cells and lymphocyte counts are part of the standard complete blood count, and are likely sufficient for the present purpose. While this would require an adaptation of the adaptive control algorithm, which is now focused on CTL data, this is easily implemented.

Such a high frequency of measurements might not be realistic in clinical practice. The classification of infected hosts based on two early measurements and attribution of optimized treatment schedules for these classes of infected hosts are more realistic. The frequency of viral load and cell count measurements would be reduced to the extent that still allows for the monitoring of treatment success 
and maintaining the benefits of the drug dose schedule generated by the adaptive control engineering. Here, we showed that the proposed adaptive strategies are robust against the reduction in the frequency of measurements. However, as the drug update frequency decreased, the adaptive strategies gradually lost their optimality in terms of larger $\mathrm{S}_{\mathrm{D}}$. This is due to the fact that, while the drug dose was administered every 12 hours, the drug dose was updated less frequently. To optimize drug consumption, we assume the same frequency for both the drug administration and the drug dose update (Supplementary Fig. S9). For each frequency, the parameters of the control-based drug dose unit (Fig. 1) are redesigned. Now, the control-based drug dose calculation unit is able to optimize (minimize) $\mathrm{S}_{\mathrm{D}}$, whereas it provides the bigger virological efficacy $\Gamma$ compared to the common clinical practice. Despite these promising results, we believe that a more efficient and intelligent algorithm could reduce the required frequency of measurements.

Pharmacokinetics of oseltamivir are best captured by a two-compartment model with one compartment for the pro-drug, oseltamivir phosphate distribution and one for its active-drug metabolite, oseltamivir carboxylate distribution (Wattanagoon et al., 2009; Davies, 2010; Canini et al., 2014). So far, we used the one-compartment approximation of the PK model without considering an oseltamivir absorption phase. To check the robustness of the adaptive strategies against delay in the oseltamivir absorption, we delayed the administration of the calculated adaptive dose by one hour (Supplementary Fig. S10). Similarly, in the common clinical practice, the fixed dose was administered at each time instant plus one hour delay. Compared to the corresponding results without delay (Fig. 2), the adaptive treatment strategies preserve their performance even in the presence of the drug absorption phase. The only difference is that the adaptive treatment strategies select higher doses to compensate for the delay. If we consider that during the absorption time, some portion of the drug is cleared from the bloodstream, the adaptive strategies still preserve their superiority to the common clinical practice (Supplementary Fig. S11). These results can be quantitatively compared in Table S3. Beside this promising result, following the general theories in Appendix A.1 one can derive the drug dose updating rule (A.5) for any other PK model satisfying the assumptions of Theorem 2. In particular, the case of a two-compartment PK model is sketched in Appendix A.3.

Note that our results cannot be directly interpreted for clinical application as model (1) was fitted to murine data. This is a limitation of our study. Switching from murine to other data may change the values of estimated parameters in (1) or the model structure. In both cases, it is possible to calculate the new drug dose updating rule. Therefore, the proposed algorithm and the presented results are limited neither to the applied murine data nor to the IAV-immune model (1) and may be applied to human data and other disease models in the future.

Less immune involvement (i.e., the reduced CTL count) under the controlbased treatment (compared to the common clinical practice) might have secondary effects on immune memory. The exact implications cannot be investigated by the current model which ignores the acquisition of immune memory. However, 
thinking of clinical application of the optimizer, it might be important to stratify patients according to their immune memory state before treatment.

In the treatment with neuraminidase inhibitors, there is a potential risk for the emergence of virus resistance which refers to the decreased susceptibility of the virus to inhibitors (Roberts, 2001; Ward et al., 2005; Aoki et al., 2007). Increasing the use of neuraminidase inhibitors, stopping the treatment prematurely after resolving the symptoms, and a low dose of the drug increase the risk of drug resistance (Canini et al., 2014; Moscona, 2005). Thus, the rational use of neuraminidase inhibitors is essential to preserve the optimality of treatment (Nitsch-Osuch and LB, 2014).

If the approved minimum dose of oseltamivir for resistance prevention was known, we could modify the proposed algorithm of drug calculation to be bounded below by the approved minimum dose. To handle the resistance issue, another idea is to enrich the model by including drug-resistant virus. This paves the way for future work on disentangling the mechanism underlying the resistance onset and how low drug dose induces resistance.

To translate the obtained results into the clinic, one issue would be the feasibility of administrating the optimal dose. The proposed algorithm though satisfying the approved upper bound for oseltamivir dosing (World Health Organization, 2009), the dose amount is not constrained to fit an available capsule size/suspension concentration. One solution is to enrich the algorithm to accommodate this constraint while another is to approximate the obtained dose to the nearest available dose amount. The second method, though less optimal, paves the way toward the application of the current results in the clinic. However, the simplest solution would be to provide capsules with very small doses and thus allow flexible dosage regimes.

As mentioned above, the described strategy is not yet ready to enter clinical reality. However, the final rule (A.5) with (A.10) to calculate the optimal dose as derived in Appendix A needs to be complemented by a user interface (GUI), which would correspond to the control-based drug dose unit in Fig. 1. The physician would then just enter the measured viral load and CTL count and receive back the optimal drug.

\section{Conclusions}

$\mathrm{PK} / \mathrm{PD}$ of the neuraminidase inhibitor oseltamivir together with the Influenza A virus modeling dynamics can potentially improve the quantitative understanding of the relationship between drug exposure efficacy and safety schedule drug guidelines in acute influenza infections. The proposed feedback control techniques can be employed to tailor drug concentrations for infected hosts depending on their responsiveness to treatment. The feedback strategies significantly improve the virological efficacy index under the new control-based treatment strategy. Quantification of the virological efficacy showed that treatment with adaptive control-based drug scheduling used about the same drug amount as in common clinical practice but with a higher virological efficacy of $79 \%$ instead of $71 \%$. When limiting the adaptive dose to $75 \mathrm{mg}$, the virological 
efficacy is back to the level of common clinical practice but using only half of the drugs.

\section{Acknowledgments}

GM was supported by the German Federal Ministry of Education and Research within the Measures for the Establishment of Systems Medicine, project SYSIMIT (BMBF-FKZ: 01ZX1608B). EAHV was supported by the Alfons und Gertrud Kassel- Stiftung. MMH was supported by the Helmholtz Initiative on Personalized Medicine - iMed; by the Human Frontier Science Program (RGP0033/2015); by the pilot project Information \& Data Science of the Helmholtz Association, project Reduced Complexity Models; by the Initiative and Networking Fund of the Helmholtz Association on the Zukunftsthemen "Aging and Metabolic Programming" (AMPro) and "Immunology and Inflammation" (ZT-0027).

\section{Appendix A. Drug dose calculation based on impulsive feedback lin- earization method}

The formula to calculate the drug dose is derived here. We introduce the required mathematical and control engineering bases and definitions. The intuitions behind definitions are briefly explained. For detailed explanations see (Khalil, 2002; Yang, 2001). First, concepts such as accessibility, relative degree and feedback linearization for a general, nonlinear model are presented in Appendix A.1. Then, the obtained results are applied to the IAV-immune model with two PK models in Appendix A.2 and A.3.

\section{Appendix A.1. Impulsive feedback linearization method}

In this study, we focus on nonlinear impulsive systems. The necessary mathematical tools to study these systems can be found in (Lakshmikantham et al., 1989). Consider a general nonlinear impulsive control system

$$
\begin{cases}\dot{X}=f(X), & t \neq \tau_{k} \\ \Delta \mathrm{X}\left(\tau_{\mathrm{k}}\right)=\mathrm{g}\left(\mathrm{X}\left(\tau_{\mathrm{k}}\right)\right) \mathrm{U}\left(\tau_{\mathrm{k}}\right), & t=\tau_{k}, k \in \mathbb{N} \\ X\left(t_{0}^{+}\right)=X\left(t_{0}\right), & \end{cases}
$$

where the vector $X \in \mathbb{R}^{n}$ consists of the system states. $f(X)$ and $g(X)$ are $n$-dimensional vectors with $n$ elements $f(X)=\left(f_{1}(X), f_{2}(X), \ldots, f_{n}(X)\right)^{T}$ and $g(X)=\left(g_{1}(X), g_{2}(X), \ldots, g_{n}(X)\right)^{T}$. Each element of $f(X)$ and $g(X)$ (i.e. $f_{i}(X)$ and $\left.g_{i}(X)\right)$ is a function of $X$ defined on $\mathbb{R}^{n}$. Let $f_{i}(X)$ and $g_{i}(X)$ be continuously differentiable functions. A function is said to be continuously differentiable if its derivatives exist and are themselves continuous functions. Thus, the function space is a set of all real-valued continuously differentiable functions $f_{i}(X)$ and 
$g_{i}(X)$ defined on $\mathbb{R}^{n} \cdot t_{0}$ is the initial time, $\mathbb{N}=\{1,2,3, \ldots\}$ and the impulsive control input $U\left(\tau_{k}\right)$ is defined as

$$
U\left(\tau_{k}\right)= \begin{cases}0, & t \neq \tau_{k} \\ u\left(\tau_{k}\right), & t=\tau_{k}\end{cases}
$$

with the scalar $u \in \mathbb{R}$. Another alternative form to represent the impulsive system (A.1) is: $\dot{X}=f(X)+g\left(X\left(\tau_{k}\right)\right) u(t) \delta\left(t-\tau_{k}\right)$, where $\delta$ is the Dirac delta function. We consider $Y=h(X)$ as the output for calculation of the control input $u$.

Before selecting a proper method to design a control input (here, the drug dose), we should check whether a considered system is controllable. Controllability means the possibility of forcing a system to move from a state $X_{1}$ to another state $X_{2}$. If a system is not controllable, we cannot force the system to show the desired behavior by applying the control input. Similar to controllability in linear systems, for nonlinear systems we have accessibility. For its definition, we first start with the concept of impulsive reachability.

Definition 1. (Rivadeneira and Moog, 2012): Starting from the initial state $X\left(t_{0}\right)$, the system (A.1) can reach the state $X\left(t_{1}\right)$ if there is a sequence of control inputs $U=\left\{u\left(\tau_{k}\right)\right\}$ applied at times $\tau_{1}<\tau_{2}<\ldots<\tau_{k}=t_{1}$, so that $X\left(X\left(t_{0}\right), U, t_{1}^{+}\right)=X\left(t_{1}\right)$. Then, $X\left(t_{1}\right)$ is the impulsively reachable state from $X\left(t_{0}\right)$.

Considering Definition 1 , let $\Omega_{k}$ denotes the set of points in $\mathbb{R}^{k}$ that can be reached by $\left(Y, \dot{Y}, \ldots, Y^{k-1}\right)^{T}$, where $Y^{k-1}$ means the $k-1^{\text {th }}$ derivative of $Y$, from $\left(h\left(X\left(t_{0}\right)\right), L_{f} h\left(X\left(t_{0}\right)\right), \ldots, L_{f}^{k-1} h\left(X\left(t_{0}\right)\right)\right)^{T}$ under $k$ control impulses at times $\tau_{1}<\tau_{2}<\ldots<\tau_{k}$. Note that $L_{f} h$ is the Lie derivative of $h$ along $f$, i.e., $L_{f} h=\frac{\partial h}{\partial X} f$ and $L_{f}^{k-1} h=\frac{\partial^{k-1} h}{\partial X} f$.

Note that while $k$ is the number of impulses in Definition 1 , in $\mathbb{R}^{k}$ it signifies the dimension of the real coordinate space. In the set $\Omega_{k}$, we put $k$ as the subscript to highlight that the set members (or points) belong to $\mathbb{R}^{k}$. Thus, the number of impulses $k$ determines the dimension of the real coordinate space to which $\Omega_{k}$ belongs. $k$ will be calculated later in Definition 2 .

Definition 1 explains only the concept of impulsively reachable state. However, to calculate the reachable state (space), first, we should know the concept of relative degree. This notion is defined in Definition 2.

Definition 2. (Khalil, 2002): The system (A.1) is said to have a relative degree $\rho(1 \leqslant \rho \leqslant n)$ if: $L_{g} L_{f}^{i-1} h(X)=0, \quad i=1,2, \ldots, \rho-1$ and $L_{g} L_{f}^{\rho-1} h(X) \neq 0$.

The impulsive relative degree $d^{0}$ is defined to be the smallest number $\rho$ of impulses required so that $\Omega_{\rho}$ contains a locally dense subset of $\mathbb{R}^{\rho}$, i.e., $d^{0}=\min \left\{\rho \in \mathbb{N} \mid L_{g} L_{f}^{\rho-1} h(X) \neq 0\right\}$. It means that, the output $Y=h(X)$ of 
system (A.1) with the impulsive relative degree $d^{0}$ is affected by the input after at least the $d^{0 t h}$ impulse.

Using the definition of relative degree, now we can explicitly calculate the reachable space as follows: if the system (A.1) with the $Y=h(X)$ has the relative degree $\rho$ on $\mathcal{N}_{1} \subset \mathbb{R}^{\rho}$, then $\mathcal{N}_{1}$ is a reachable space. Thus, the reachable space is the space belonging to $\mathbb{R}^{\rho}$ on which the system has the relative degree $\rho$.

Based on the previous definitions and using the Lie bracket $[f, g]$ or $a d_{f} g$ as $[f, g]=a d_{f} g=\frac{\partial g}{\partial X} f-\frac{\partial f}{\partial X} g$, now we can define the accessibility property.

Theorem 1. (Rivadeneira and Moog, 2012): The system (A.1) with the relative degree $\rho$ is said to be impulsively accessible on $\mathcal{N}_{2} \subset \mathbb{R}^{\rho},\left(\mathcal{N}_{2} \subset \mathcal{N}_{1}\right)$ if dimension of involutive closure (Isidori, 1995) of $\left\{g, a d_{f} g, \ldots, a d_{f}^{\rho-1} g\right\}$ is $\rho$, i.e., $\operatorname{dim} \overline{\left\{g, a d_{f} g, \ldots, a d_{f}^{\rho-1} g\right\}}=\rho$, where

$a d_{f}^{j} g=\left[f, a d_{f}^{j-1} g\right], j \geqslant 1$, and $a d_{f}^{0} g=g$.

Assume the considered system satisfies the accessibility criterion. Then, we can design a proper control input for the system. While for linear impulsive systems many theories have been developed so far, for nonlinear impulsive systems less advanced theories exist. Thus, one idea would be to linearize the original nonlinear model. However, instead of the linear approximation, a better idea is to recast a nonlinear model as a linear one by considering a coordinate transformation. The transformation is selected in a way that the original nonlinear model is seen linearly in the new coordinate. This method is called feedback linearization (Khalil, 2002).

In order to apply the feedback linearization method to the impulsive systems, first we need to find a diffeomorphism $Z=\phi(X)$ and an impulsive state feedback $u\left(\tau_{k}\right)$ to transform the nonlinear system into a linear one.

The diffeomorphism $Z=\left(z_{1}, z_{2}, \ldots, z_{\rho}\right)^{T}$ with

$$
z_{i}=L_{f}^{i-1} h(X), \quad i=1,2, \ldots, \rho,
$$

and $L_{f}^{0} h(X)=h(X)$, results in

$$
\left\{\begin{array}{l}
\dot{z}_{i-1}=z_{i}, \quad i=1,2, \ldots, \rho-1 \\
\dot{z}_{\rho}=\beta(X)+\alpha(X) U\left(\tau_{k}\right)
\end{array}\right.
$$

where $\beta=L_{f}^{\rho} h(X), \alpha=L_{g} L_{f}^{\rho-1} h(X)$ and $U\left(\tau_{k}\right)$ is defined as (A.2).

To remove the nonlinear terms $\beta(X)$ and $\alpha(X)$ from (A.4), the following impulsive state feedback control law is chosen

$$
u\left(\tau_{k}\right)=\frac{1}{\alpha(X)}\left(v\left(\tau_{k}\right)-\beta(X)\right)
$$


which leads to the following linear impulsive system

$$
\begin{cases}\dot{Z}=A Z, & t \neq \tau_{k} \\ \Delta \mathrm{Z}=\mathrm{B} v\left(\tau_{\mathrm{k}}\right), & t=\tau_{k}, k \in \mathbb{N}\end{cases}
$$

where $A=\left(\begin{array}{ccccc}0 & 1 & 0 & \cdots & 0 \\ 0 & 0 & 1 & \cdots & 0 \\ \vdots & \vdots & \vdots & \ddots & \vdots \\ 0 & 0 & 0 & \cdots & 1 \\ 0 & 0 & 0 & 0 & 0\end{array}\right)$, and $B=\left(\begin{array}{c}0 \\ 0 \\ \vdots \\ 0 \\ 1\end{array}\right)$ at each $t=\tau_{k}$.

In fact, in the new coordinate (A.3) with the impulsive feedback control (A.5), the nonlinear impulsive control system (A.1) is seen linearly as (A.6). The last step is to use the pole placement method for the linear impulsive control system (Yang, 2001) to choose the coefficients $K=\left(k_{1}, k_{2}, \ldots, k_{\rho}\right)$ of $v\left(\tau_{k}\right)=K Z\left(\tau_{k}\right)$.

If the system (A.1) has the relative degree $\rho=n$, then (A.1) can be fully transformed to (A.6). For $\rho<n$, the other $n-\rho$ states are not seen in the input-output channel and can be represented as $\dot{\eta}=q(Z, \eta), \eta \in \mathbb{R}^{n-\rho}$. $\eta$ 's are called internal dynamics. In this case, the control input $u$ can force only $z_{i}, i=1,2, \ldots, \rho$ to converge to zero. If zero dynamics, obtained by setting $Z=0$ in the $\dot{\eta}$ equation, i.e., $\dot{\eta}=q(0, \eta)$ are asymptotically stable, then, the full system is asymptotically stable (Khalil, 2002). Theorem 2 summarizes the results.

Theorem 2. Suppose the system (A.1) with $X, f, g \in \mathbb{R}^{n}, u \in \mathbb{R}$ and $Y=h(X)$ as an output has the relative degree $\rho$ and satisfies the accessibility criterion. Let the system admit asymptotically stable zero dynamics. Then, the system (A.1) is asymptotically stable under the control input (A.5).

In Appendix A.2, details of applying Theorem 2 to stabilize the IAV-immune model (1)-(4) are described.

Appendix A.2. Application of impulsive feedback linearization method to the drug dosing problem using an approximated PK model of oseltamivir

As previously introduced, the IAV-immune model under oseltamivir treatment (1)-(4) is in the framework of nonlinear impulsive control systems. Nonlinearity originates from the structure of the IAV-immune model. The impulsive property is due to the drug dynamics which are continuous most of the time (i.e., $t \neq \tau_{k}$ ), but at some time instants (i.e., $t=\tau_{k}$ ) when the drug is administered, we have sudden changes in the drug dynamics.

The model (1) has a nontrivial steady-state $\left(T_{0}, 0\right)$, where $T_{0}=\frac{S_{E}}{c_{e}}$. To simplify the next steps of the analysis, we shift the steady-state to the origin by the change of variables as $(\bar{E}, \bar{V})=\left(E-T_{0}, V\right)$. The resulting transformed system to the origin (including the drug dynamics) can be represented in the 
general form (A.1) with $X \triangleq(\bar{E}, \bar{V}, D)^{T}$ and vectors

$$
\begin{aligned}
& f(X)=\left(\begin{array}{c}
r\left(\bar{E}+T_{0}\right)\left(\frac{\bar{V}}{\bar{V}+k_{e}}\right)-c_{e} \bar{E} \\
p(1-\epsilon) \bar{V}\left(1-\frac{\bar{V}}{k_{v}}\right)-c_{v}\left(\bar{E}+T_{0}\right) \bar{V} \\
-\gamma D
\end{array}\right), \\
& g(X)=(0,0,1)^{T} .
\end{aligned}
$$

We consider $Y=h(X)=\bar{V}$ as the output for calculation of the control-based drug dose $u$. First, we calculate the relative degree of system (A.1) with (A.7). Using Definition 2 we have

$$
\begin{aligned}
& i=1, \quad L_{g} h=\frac{\partial h}{\partial X} g=(0,1,0)(0,0,1)^{T}=0 \\
& i=2, \quad L_{g} L_{f} h=\frac{\partial L_{f} h}{\partial X} g= \\
& \left(-c_{v} \bar{V},-c_{v}\left(\bar{E}+T_{0}\right)+p(1-\epsilon)\left(1-\frac{2 \bar{V}}{k_{v}}\right),-\frac{D_{50} p \bar{V}}{\left(D+D_{50}\right)^{2}}\left(1-\frac{\bar{V}}{k_{v}}\right)\right) \times \\
& (0,0,1)^{T}=-\frac{D_{50} p \bar{V}}{\left(D+D_{50}\right)^{2}}\left(1-\frac{\bar{V}}{k_{v}}\right) .
\end{aligned}
$$

So, the impulsive relative degree is $\rho=2$. This means that the impulsive input affects the output after at least the second impulse. In addition, the strictly positive subspace of $\mathbb{R}^{2}$, i.e. $\mathbb{R}_{+}^{2}$, is the reachable space.

To check the accessibility, from Theorem 1, we have $a d_{f} g=\frac{\partial g}{\partial X} f-\frac{\partial f}{\partial X} g=$ $\left(0, \frac{D_{50} p \bar{V}}{\left(D+D_{50}\right)^{2}}\left(1-\frac{\bar{V}}{k_{v}}\right), \gamma\right)^{T}$. Thus, the dimension of $\overline{\left\{g, a d_{f}^{g}\right\}}$ is equal to 2 and this shows $\mathbb{R}_{+}^{2}$ is accessible.

To transfer the system (A.1) to the linear impulsive system, we define the diffeomorphism

$$
\begin{aligned}
& z_{1}=h=\bar{V} \\
& z_{2}=L_{f} h=p(1-\epsilon) \bar{V}\left(1-\frac{\bar{V}}{k_{v}}\right)-c_{v}\left(\bar{E}+T_{0}\right) \bar{V}
\end{aligned}
$$

Finally, the designed drug dose (impulsive control input) is calculated based on (A.5) with 


$$
\begin{aligned}
\alpha= & -\frac{d_{50} p \bar{V}}{\left(d+d_{50}\right)^{2}}\left(1-\frac{\bar{V}}{k_{v}}\right) \\
\beta=- & c_{v} \bar{V} \dot{\bar{E}}+\left(-c_{v}\left(\bar{E}+T_{0}\right)+p(1-\epsilon)\left(1-\frac{2 \bar{V}}{k_{v}}\right)\right) \dot{\bar{V}} \\
& +\frac{D_{50} \gamma p d \bar{V}}{\left(D+D_{50}\right)^{2}}\left(1-\frac{\bar{V}}{k_{v}}\right)
\end{aligned}
$$

The designed $u$ (A.5) with (A.10) forces $z_{1}$ and $z_{2}$ to converge to zero asymptotically. In addition, the origin of the zero dynamics $\dot{\bar{E}}=-c_{e} \bar{E}$ is exponentially stable.

Appendix A.3. Application of impulsive feedback linearization method to the drug dosing problem using a two-compartment PK model of oseltamivir

To show that the theories introduced in Appendix A.1 are general and can be applied to other PK models, here, we considered a more realistic PK model of oseltamivir.

The two-compartment PK model of oseltamivir in (Wattanagoon et al., 2009; Davies, 2010; Canini et al., 2014) with one compartment for pro-drug, oseltamivir phosphate (OP) distribution and one compartment for its active-drug metabolite, oseltamivir carboxylate (OC) distribution reads as

$$
\begin{aligned}
& \left\{\begin{array}{l}
\dot{G}=-k_{a} G, \\
O \dot{P}=k_{a} G-k_{f} O P, \\
O \dot{C}=k_{f} O P-k_{e l} O C,
\end{array}\right. \\
& \Delta \mathrm{G}\left(\tau_{k}\right)=\mathrm{G}\left(\tau_{k}^{+}\right)-\mathrm{G}\left(\tau_{k}^{-}\right)=u\left(\tau_{k}\right), \quad k=1,2, \ldots
\end{aligned}
$$

where $\mathrm{G}$ is the depot compartment out of which the drug is absorbed. In the general framework (A.1), we have

$$
\begin{aligned}
& X=(\bar{E}, \bar{V}, G, O P, O C)^{T}, \\
& f(X)=\left(\begin{array}{c}
r\left(\bar{E}+T_{0}\right)\left(\frac{\bar{V}}{\bar{V}+k_{e}}\right)-c_{e} \bar{E} \\
p\left(1-\frac{O C}{O C+O C_{50}}\right) \bar{V}\left(1-\frac{\bar{V}}{k_{v}}\right)-c_{v}\left(\bar{E}+T_{0}\right) \bar{V} \\
-k_{a} G \\
k_{a} G-k_{f} O P \\
k_{f} O P-k_{e l} O C
\end{array}\right), \\
& g(X)=(0,0,1,0,0)^{T} .
\end{aligned}
$$

Compared to (A.7), the model dimension is increased from 3 to 5 . The next step is computing the relative degree (Definition 2) and checking the accessibility criterion (Theorem 1). Considering $h=\bar{V}$, since $L_{g} L_{f}^{3} h(X) \neq 0$, Definition 2 gives the relative degree $\rho=4$. Using Theorem 1 , the accessibility criterion is 
satisfied. To compute the drug dose for the two-compartment model, according to (A.5), we need to compute $\alpha(X)$ and $\beta(X)$, which are given by

$$
\alpha(X)=\frac{\partial M}{\partial G}, \beta(X)=\frac{\partial M}{\partial \bar{E}} f_{1}+\frac{\partial M}{\partial \bar{V}} f_{2}+\frac{\partial M}{\partial G} f_{3}+\frac{\partial M}{\partial O P} f_{4}+\frac{\partial M}{\partial O C} f_{5},
$$

where

$$
\begin{aligned}
M & =\frac{\partial Q}{\partial \bar{E}} f_{1}+\frac{\partial Q}{\partial \bar{V}} f_{2}+\frac{\partial Q}{\partial O P} f_{4}+\frac{\partial Q}{\partial O C} f_{5} \\
Q & =\frac{\partial f_{2}}{\partial \bar{E}} f_{1}+\frac{\partial f_{2}}{\partial \bar{V}} f_{2}+\frac{\partial f_{2}}{\partial O C} f_{5} \\
f_{1} & =r\left(\bar{E}+T_{0}\right)\left(\frac{\bar{V}}{\bar{V}+k_{e}}\right)-c_{e} \bar{E} \\
f_{2} & =p\left(1-\frac{O C}{O C+O C_{50}}\right) \bar{V}\left(1-\frac{\bar{V}}{k_{v}}\right)-c_{v}\left(\bar{E}+T_{0}\right) \bar{V} \\
f_{3} & =-k_{a} G \\
f_{4} & =k_{a} G-k_{f} O P \\
f_{5} & =k_{f} O P-k_{e l} O C .
\end{aligned}
$$

Note that, $\frac{\partial \Theta}{\partial Z}$ means the partial derivative of $\Theta,\left(\Theta=M, Q, f_{2}\right)$ with respect to $Z,(Z=\bar{E}, V, G, O P, O C)$.

Thus, defining the updating rule of oseltamivir dose is straightforward following the theories of Appendix A.1. As the dimension of the system to be controlled (i.e., the dimension of the IAV-immune or the PK model) increases, calculation of the optimal dose will be computationally complicated but still possible. 


\section{Supplementary material}

Eleven figures and three tables are presented in Supplementary Material.

Figure S1: High dose vs low dose control-based treatment started on day 3 after infection. a) Immune response, b) viral load and c) drug dynamics. Adaptive dosing with a higher total amount of drug results in faster viral clearance time and lower number of effector cells.

Figure S2: Low dose oseltamivir treatments started on day 2 after infection. a) Immune response, b) viral load and c) drug dynamics under the three different drug administration strategies for the low dose common clinical practice with $\Delta \mathrm{D}_{\text {fixed }}=75 \mathrm{mg}$ and imposing $\Delta \mathrm{D}_{\text {adaptive }} \leq 75$ $\mathrm{mg}$ in the safe control-based treatment. For earlier diagnosis, the adaptive strategies choose a higher amount of the drug to compensate for the weak immune response.

Figure S3: Low dose oseltamivir treatments started on day 4 after infection. a) Immune response, b) viral load and c) drug dynamics under the three different drug administration strategies for the low dose common clinical practice with $\Delta \mathrm{D}_{\text {fixed }}=75 \mathrm{mg}$ and imposing $\Delta \mathrm{D}_{\text {adaptive }} \leq 75$ $\mathrm{mg}$ in the safe control-based treatment. For late diagnosis, the adaptive strategies benefit from the stronger immune response and reduce the total administered drug.

Figure S4: Variation of parameters and initial conditions taken from a uniform distribution. Histograms of random parameters/initial conditions in Monte Carlo analysis used to generate the results in figures 4 and S5. All parameters in the IAV-immune model (1)-(4) and the initial states $V(0)$ and $E(0)$ are selected uniformly from the interval $[\bar{m}-20 \% \bar{m}, \bar{m}+20 \% \bar{m}]$, where $\bar{m}$ is the median value of $m$ with $m \in\left\{r, k_{e}, c_{e}, p, k_{v}, c_{v}, D_{50}, \gamma, V(0), E(0)\right\}$.

Figure S5: Monte Carlo simulations (with uniform distribution) of high dose treatments. Histograms of virological efficacy index $\Gamma$ and total administered drug (area under the drug curve $\mathrm{S}_{\mathrm{D}}$ ) of Monte Carlo analysis reflecting the diversity of infected hosts. The different treatment strategies are shown with the same color code and line style as before. Treatment was started on day 2,3 , or 4 in panels $(\mathrm{a}, \mathrm{b}),(\mathrm{c}, \mathrm{d})$, and $(\mathrm{e}, \mathrm{f})$, respectively. Simulations were performed with $\Delta \mathrm{D}_{\text {fixed }}=150 \mathrm{mg}$ for the low dose common clinical practice and with $\Delta D_{\text {adaptive }} \leq 150 \mathrm{mg}$ for the safe control-based treatment. $(*)$ denotes the statistical significance between different treatments based on the one-way ANOVA test ( $\mathrm{p}$-value $<\frac{0.05}{3}$ ). The control-based treatment in $\mathrm{a}, \mathrm{c}, \mathrm{e}$ and the safe control-based treatment in $\mathrm{b}, \mathrm{d}, \mathrm{f}$ show better performances compared to the common clinical practice.

Figure S6: Variation of parameters and initial conditions taken from a normal distribution. Histograms of random parameters/initial conditions in Monte Carlo analysis used to generate the results in figures S7 and S8. All parameters in the IAV-immune model (1)-(4) and the initial states $V(0)$ and $E(0)$ are selected from a normal distribution with median $\bar{m}$ and standard deviation $0.05 \bar{m}$. 
Figure S7: Monte Carlo simulations (with normal distribution) of low dose treatments. Histograms of virological efficacy index $\Gamma$ and total administered drug (area under the drug curve $\mathrm{S}_{\mathrm{D}}$ ) of Monte Carlo analysis reflecting the diversity of infected hosts. The different treatment strategies are shown with the same color code and line style as before. Treatment was started on day 2,3 , or 4 in panels $(\mathrm{a}, \mathrm{b}),(\mathrm{c}, \mathrm{d})$, and $(\mathrm{e}, \mathrm{f})$, respectively. Simulations were performed with $\Delta \mathrm{D}_{\text {fixed }}=75 \mathrm{mg}$ for the low dose common clinical practice and with $\Delta \mathrm{D}_{\text {adaptive }} \leq 75 \mathrm{mg}$ for the safe control-based treatment. $(*)$ denotes the statistical significance between different treatments based on the one-way ANOVA test ( $\mathrm{p}$-value $<\frac{0.05}{3}$ ). The control-based treatment in a,c,e and the safe control-based treatment in b,d,f show better performances compared to the common clinical practice. Similar to the results with the uniform distribution, the improved performance of the adaptive strategies is preserved.

Figure S8: Monte Carlo simulations (with normal distribution) of high dose treatments. Histograms of virological efficacy index $\Gamma$ and total administered drug (area under the drug curve $\mathrm{S}_{\mathrm{D}}$ ) of Monte Carlo analysis reflecting the diversity of infected hosts. The different treatment strategies are shown with the same color code and line style as before. Treatment was started on day 2,3 , or 4 in panels $(\mathrm{a}, \mathrm{b}),(\mathrm{c}, \mathrm{d})$, and $(\mathrm{e}, \mathrm{f})$, respectively. Simulations were performed with $\Delta \mathrm{D}_{\text {fixed }}=150 \mathrm{mg}$ for the low dose common clinical practice and with $\Delta \mathrm{D}_{\text {adaptive }} \leq 150 \mathrm{mg}$ for the safe control-based treatment. $(*)$ denotes the statistical significance between different treatments based on the one-way ANOVA test ( $\mathrm{p}$-value $<\frac{0.05}{3}$ ). The control-based treatment in a,c,e and the safe control-based treatment in b,d,f show better performances compared to the common clinical practice. Similar to the results with the uniform distribution, the improved performance of the adaptive strategies is preserved.

Figure S9: Low dose oseltamivir treatments (started on day 3 after infection) with different time intervals of drug administration. Histograms of a) virological efficacy index $\Gamma$ and b) total administered drug (area under the drug curve $\mathrm{S}_{\mathrm{D}}$ ) for the common clinical practice and the control-based treatment with the low dose. Unlike in Fig. 5, here, we assume the same time interval for both drug administration and drug dose update. For each time interval, the control-based treatment highlights its performance by showing the higher virological efficacy $\Gamma$ while using a lower amount of the drug.

Figure S10: Low dose oseltamivir treatments (started on day 3 after infection) considering one hour delay in the oseltamivir absorption. a) Immune response, b) viral load and c) drug dynamics under the three different drug administration strategies for the low dose common clinical practice with $\Delta \mathrm{D}_{\text {fixed }}=75 \mathrm{mg}$ and imposing $\Delta \mathrm{D}_{\text {adaptive }} \leq 75 \mathrm{mg}$ in the safe control-based treatment. We assume that each drug dose becomes effective with one hour delay from the time of administration. Compared to the corresponding results without delay (Fig. 2), the adaptive treatment strategies preserve their performance even in the presence of the drug absorption phase. The only difference is that here, the adaptive treatment strategies select higher doses to compensate for the considered delay. This in turn leads to the faster viral clearance.

Figure S11: Low dose oseltamivir treatments (started on day 3 after infection) considering drug elimination during the absorption time. a) Immune response, b) viral load and c) drug dynamics under the three different drug administration strategies. We assume that $2 / 3$ of each drug dose becomes effective with one hour delay from the time of administration and the remaining drug is cleared from the bloodstream. Compared to the corresponding results without considering the drug elimination (Fig. S10), the drug doses decrease and this leads to a slower viral load clearance. The safe control-based treatment selects small (non-zero) doses from day 7 but it can still achieve the same virological efficacy as in the common clinical practice (see Table S3). 
Table S1: Median values of Monte Carlo simulations with uniform distribution. Comparison of the virological efficacy index $\Gamma$ and the total amount of administered oseltamivir (area under the drug curve $\mathrm{S}_{\mathrm{D}}$ ) for the three different treatments (under low and high dose condition) and at different treatment starting days.

\begin{tabular}{llllllll}
\multicolumn{8}{c}{$\Delta D_{\text {adaptive }} \leq \Delta \mathrm{D}_{\text {fixed }}=75 \mathrm{mg}$} \\
\hline \hline Starting day of treatments & \multicolumn{2}{c}{ day 2} & \multicolumn{2}{c}{ day 3} & \multicolumn{2}{c}{ day 4} \\
\hline Treatment strategies & $\Gamma$ & $\mathrm{S}_{\mathrm{D}}$ & $\Gamma$ & $\mathrm{S}_{\mathrm{D}}$ & $\Gamma$ & $\mathrm{S}_{\mathrm{D}}$ \\
\hline Common clinical practice & $90 \%$ & 305 & $65 \%$ & 305 & $31 \%$ & 305 \\
Control-based treatment & $95 \%$ & 382 & $71 \%$ & 185 & $33 \%$ & 103 \\
Safe control-based treatment & $90 \%$ & 194 & $65 \%$ & 146 & $31 \%$ & 88 \\
\hline
\end{tabular}

$$
\Delta \mathrm{D}_{\text {adaptive }} \leq \Delta \mathrm{D}_{\text {fixed }}=150 \mathrm{mg}
$$

\begin{tabular}{lllllll}
\hline \hline Starting day of treatments & \multicolumn{2}{c}{ day 2} & \multicolumn{2}{c}{ day 3} & \multicolumn{2}{c}{ day 4} \\
\hline Treatment strategies & $\Gamma$ & $\mathrm{S}_{\mathrm{D}}$ & $\Gamma$ & $\mathrm{S}_{\mathrm{D}}$ & $\Gamma$ & $\mathrm{S}_{\mathrm{D}}$ \\
\hline Common clinical practice & $95 \%$ & 609 & $74 \%$ & 609 & $36 \%$ & 609 \\
Control-based treatment & $97 \%$ & 498 & $76 \%$ & 400 & $38 \%$ & 369 \\
Safe control-based treatment & $96 \%$ & 404 & $74 \%$ & 357 & $36 \%$ & 334 \\
\hline
\end{tabular}

Table S2: Median values of Monte Carlo simulations with normal distribution. Comparison of the virological efficacy index $\Gamma$ and the total amount of administered oseltamivir (area under the drug curve $\mathrm{S}_{\mathrm{D}}$ ) for the three different treatments (under low and high dose condition) and at different starting days of treatments.

$\Delta \mathrm{D}_{\text {adaptive }} \leq \Delta \mathrm{D}_{\text {fixed }}=75 \mathrm{mg}$

\begin{tabular}{lllllll}
\hline \hline Starting day of treatments & \multicolumn{2}{c}{ day 2} & \multicolumn{2}{c}{ day 3} & \multicolumn{2}{c}{ day 4} \\
\hline Treatment strategies & $\Gamma$ & $\mathrm{S}_{\mathrm{D}}$ & $\Gamma$ & $\mathrm{S}_{\mathrm{D}}$ & $\Gamma$ & $\mathrm{S}_{\mathrm{D}}$ \\
\hline Common clinical practice & $93 \%$ & 320 & $72 \%$ & 320 & $38 \%$ & 320 \\
Control-based treatment & $97 \%$ & 545 & $79 \%$ & 324 & $43 \%$ & 223 \\
Safe control-based treatment & $93 \%$ & 241 & $72 \%$ & 159 & $38 \%$ & 125 \\
\hline
\end{tabular}

$\Delta \mathrm{D}_{\text {adaptive }} \leq \Delta \mathrm{D}_{\text {fixed }}=150 \mathrm{mg}$

\begin{tabular}{lllllll}
\hline \hline Starting day of treatments & \multicolumn{2}{c}{ day 2} & \multicolumn{2}{c}{ day 3} & \multicolumn{2}{c}{ day 4} \\
\hline Treatment strategies & $\Gamma$ & $\mathrm{S}_{\mathrm{D}}$ & $\Gamma$ & $\mathrm{S}_{\mathrm{D}}$ & $\Gamma$ & $\mathrm{S}_{\mathrm{D}}$ \\
\hline Common clinical practice & $97 \%$ & 640 & $80 \%$ & 640 & $44 \%$ & 640 \\
Control-based treatment & $98 \%$ & 607 & $82 \%$ & 583 & $47 \%$ & 525 \\
Safe control-based treatment & $97 \%$ & 463 & $80 \%$ & 427 & $44 \%$ & 399 \\
\hline
\end{tabular}


Table S3: Low dose oseltamivir treatments (started on day 3 after infection) considering one hour delay in the drug absorption/and elimination of some portion of the drug during the absorption time. Comparison of the virological efficacy index $\Gamma$ and total administered drug (area under the drug curve $\left.\mathrm{S}_{\mathrm{D}}\right)$ for the three different treatments under low dose condition $\left(\Delta \mathrm{D}_{\text {adaptive }} \leq \Delta \mathrm{D}_{\text {fixed }}=\right.$ $75 \mathrm{mg}) . \Gamma$ and $\mathrm{S}_{\mathrm{D}}$ are shown with the corresponding differences to the results without delay and drug elimination (Table 3). $\Delta \chi=\mid \chi$ (with delay/and drug elimination)- $\chi$ (without delay and drug elimination) $\mid, \chi=\Gamma, \mathrm{S}_{\mathrm{D}}$. Including delay reduces $\Gamma$ in all treatment strategies by $1 \%$. Here, again the control-based treatment gains the highest $\Gamma$ followed by the other two treatment strategies with the same $\Gamma$. Regarding $\mathrm{S}_{\mathrm{D}}$, the adaptive strategies select higher doses to compensate for the drug absorption phase. Considering the drug clearance from the bloodstream during the delay leads to the reduction in $\Gamma$ and $\mathrm{S}_{\mathrm{D}}$. Again adaptive strategies preserve their superiority to the common clinical practice. Data are derived from Fig. S10 and Fig. S11.

Results with delay in the drug absorption

\begin{tabular}{lll}
\hline \hline Treatment strategies & $\Gamma(\Delta \Gamma)$ & $\mathrm{S}_{\mathrm{D}}\left(\Delta \mathrm{S}_{\mathrm{D}}\right)$ \\
\hline Common clinical practice & $70 \%(1 \% \downarrow)$ & $316(\simeq 0)$ \\
Control-based treatment & $78 \%(1 \% \downarrow)$ & $376(27 \uparrow)$ \\
Safe control-based treatment & $70 \%(1 \% \downarrow)$ & $162(7 \uparrow)$ \\
\hline
\end{tabular}

Results with drug elimination during the absorption time

\begin{tabular}{llc}
\hline \hline Common clinical practice & $61 \%(10 \% \downarrow)$ & $196(120 \downarrow)$ \\
Control-based treatment & $71 \%(8 \% \downarrow)$ & $172(177 \downarrow)$ \\
Safe control-based treatment & $61 \%(10 \% \downarrow)$ & $89 \quad(66 \downarrow)$ \\
\hline
\end{tabular}




\section{References}

Alexander, M. E., Bowman, C. S., Feng, Z., Gardam, M., Moghadas, S. M., Röst, G., Wu, J., and Yan, P., 2007. Emergence of drug resistance: implications for antiviral control of pandemic influenza. Proceedings of the Royal Society of London B: Biological Sciences 274, 1675-1684.

Aoki, F., Boivin, G., and Roberts, N., 2007. Influenza virus susceptibility and resistance to oseltamivir. Antivir. Ther. 12, 603-613.

Baccam, P., Beauchemin, C., Macken, C., Hayden, F., and Perelson, A., 2006. Kinetics of influenza A virus infection in humans. J. Virol. 80, 7590-7599.

Binnicker, M., Espy, M., Irish, C., and Vetter, E., 2015. Direct detection of influenza $\mathrm{A}$ and $\mathrm{B}$ viruses in less than 20 minutes using a commercially available rapid PCR assay. J. Clin. Microbiol. 53, 2353-2354.

Boianelli, A., Nguyen, V., Ebensen, T., Schulze, K., Wilk, E., Sharma, N., Stegemann-Koniszewski, S., Bruder, D., Toapanta, F., Guzmán, C. et al., 2015. Modeling influenza virus infection: A roadmap for influenza research. Viruses. 7, 5274-5304.

Boianelli, A., Sharma-Chawla, N., Bruder, D., and Hernandez-Vargas, E. A., 2016. Oseltamivir PK/PD modeling and simulation to evaluate treatment strategies against influenza-pneumococcus coinfection. Front. Cell. Infect. Microbiol. 6, 60 .

Canini, L., Conway, J., Perelson, A., and Carrat, F., 2014. Impact of different oseltamivir regimens on treating influenza $\mathrm{A}$ virus infection and resistance emergence: insights from a modelling study. PLoS Comput. Biol. 10, e1003568.

Cardozo, E., and Zurakowski, R., 2012. Robust closed-loop minimal sampling method for HIV therapy switching strategies. IEEE T. Bio-Med. Eng. 59, $2227-2234$.

$\begin{array}{ccccc}\text { Centers } & \text { for } & \text { Disease } & \text { Control and } & \text { Prevention, } \\ 2016 . & \text { Clinical Signs and Symptoms of Influenza. } & \text { and } \\ \text { https://www.cdc.gov/flu/professionals/acip/clinical.htm. }\end{array}$

Centers for Disease Control and Prevention, 2018. Prevention and control of seasonal influenza with vaccines: Recommendations of the Advisory Committee on Immunization Practices (ACIP). https://www.cdc.gov/flu/professionals/acip/2017-18summary.htm.

Chang, H., and Astolfi, A., 2008. Control of HIV infection dynamics. IEEE Contr. Syst. Mag. 28, 28-39.

Cobelli, C., Renard, E., and Kovatchev, B., 2011. Artificial pancreas: Past, present, future. Diabetes. 60, 2672-2682. 
Davies, B. E., 2010. Pharmacokinetics of oseltamivir: an oral antiviral for the treatment and prophylaxis of influenza in diverse populations. Antimicrob. Agents Chemother. 65, ii5-ii10.

Dhumpa, R., Tuan, T., Wang, X., Bertram, R., and Roper, M., 2014. Negative feedback synchronizes islets of Langerhans. Biophys. J. 106, $2275-2282$.

Dobrovolny, H., Gieschke, R., Davies, B., Jumbe, N., and Beauchemin, C., 2011. Neuraminidase inhibitors for treatment of human and avian strain influenza: A comparative modeling study. J. Theor. Biol. 269, 234-244.

Dobrovolny, H., Reddy, M., Kamal, M., Rayner, C., and Beauchemin, C., 2013. Assessing mathematical models of influenza infections using features of the immune response. PLoS ONE. 8, e57088.

Doyle, F., Huyett, L., Lee, J., Zisser, H., and Dassau, E., 2014. Closed-loop artificial pancreas systems: Engineering the algorithms. Diabetes Care. 37, 1191-1197.

van der Graaf, P., and Benson, N., 2011. Systems pharmacology: bridging systems biology and pharmacokinetics-pharmacodynamics (PKPD) in drug discovery and development. Pharm. Res. 28, 1460-1464.

Gubar, E., and Zhu, Q., 2013. Optimal control of influenza epidemic model with virus mutations. In 2013 European Control Conference (ECC) (pp. $3125-3130)$.

Gubareva, L., Kaiser, L., and Hayden, F., 2000. Influenza virus neuraminidase inhibitors. The Lancet. 355, 827-835.

Hancioglu, B., Swigon, D., and Clermont, G., 2007. A dynamical model of human immune response to influenza A virus infection. J. Theor. Biol. 246, 70-86.

Handel, A., Longini, I. J., and Antia, R., 2007. Neuraminidase inhibitor resistance in influenza: assessing the danger of its generation and spread. PLoS Comput. Biol. 3, e240.

He, G., Massarella, J., and Ward, P., 1999. Clinical pharmacokinetics of the prodrug oseltamivir and its active metabolite Ro 64-0802. Clinical Pharmacokinetics 37, 471-484.

Hernandez-Vargas, E., Colaneri, P., and Middleton, R., 2013. Optimal therapy scheduling for a simplified HIV infection model. Automatica. 49, $2874-$ 2880 .

Hernandez-Vargas, E., Colaneri, P., and Middleton, R., 2014a. Switching strategies to mitigate HIV mutation. IEEE T. Contr. Syst. T. 22, 1623-1628. 
Hernandez-Vargas, E., Wilk, E., Canini, L., Toapanta, F., Binder, S., Uvarovskii, A., Ross, T., Guzmán, C., Perelson, A., and Meyer-Hermann, M., 2014b. Effects of aging on influenza virus infection dynamics. J. Virol. 88, 41234131.

Holder, B., Simon, P., Liao, L., Abed, Y., Bouhy, X., Beauchemin, C., and Boivin, G., 2011. Assessing the in vitro fitness of an oseltamivir-resistant seasonal A/H1N1 influenza strain using a mathematical model. PLoS ONE. 6, e14767.

Isidori, A., 1995. Nonlinear control systems. (3rd ed.). Springer-Verlag, New York.

Itik, M., Salamci, M., and Banks, S., 2009. Optimal control of drug therapy in cancer treatment. Nonlinear Anal-Theor. 71, e1473 - e1486.

Jaberi-Douraki, M., and Moghadas, S., 2014. Optimal control of vaccination dynamics during an influenza epidemic. Math. Biosci. Eng. 11, 1045-1063.

Jacobs, P., El Youssef, J., Castle, J., Engle, J., Branigan, D., Johnson, P., Massoud, R., Kamath, A., and Ward, W., 2011. Development of a fully automated closed loop artificial pancreas control system with dual pump delivery of insulin and glucagon. In Engineering in Medicine and Biology Society, EMBC, Ann Intern Conf IEEE (pp. 397-400).

Khalil, H., 2002. Nonlinear control. Prentice Hall, New Jersey.

Kilbourne, E., 2006. Influenza pandemics of the 20th century. Emerg. Infect. Dis. $12,9-14$.

Kim, D., and Poudel, B., 2013. Tools to detect influenza virus. Yonsei. Med. J. $54,560-566$.

Kokotović, P., Khalil, H., and ÓReilly, J., 1999. Singular perturbation methods in control: Analysis and design. Philadelphia, PA: Society for Industrial and Applied Mathematics.

Lakshmikantham, V., Bainov, D. D., and Simeonov, P. S., 1989. Theory of Impulsive Differential Equations. World Scientific.

Lee, H., Topham, D., Park, S., Hollenbaugh, J., Treanor, J., Mosmann, T., Jin, X., Ward, B., Miao, H., Holden-Wiltse, J., Perelson, A., Zand, M., and Wu, H., 2009. Simulation and prediction of the adaptive immune response to influenza A virus infection. J. Virol. 83, 7151-7165.

Lee, J., Kim, J., and Kwon, H., 2013. Optimal control of an influenza model with seasonal forcing and age-dependent transmission rates. J. Theor. Biol. $317,310-320$. 
Lee, S., Chowell, G., and Castillo-Chávez, C., 2010. Optimal control for pandemic influenza: The role of limited antiviral treatment and isolation. J. Theor. Biol. 265, 136-150.

Legrand, M., Comets, E., Aymard, G., Tubiana, R., Katlama, C., and Diquet, B., 2003. An in vivo pharmacokinetic/pharmacodynamic model for antiretroviral combination. HIV Clin. Trials. 4, 170-183.

Marois, I., Cloutier, A., Garneau, É., and Richter, M. V., 2012. Initial infectious dose dictates the innate, adaptive, and memory responses to influenza in the respiratory tract. J Leukoc. Biol. 92, 107-121.

Martin, R., 1992. Optimal control drug scheduling of cancer chemotherapy. Automatica. 28, $1113-1123$.

Martin, R., and Teo, K., 1993. Optimal control of drug administration in cancer chemotherapy. Singapore: World Scientific.

McDonagh, M., and Bell, E., 1995. The survival and turnover of mature and immature CD8 T cells. Immunology. 84, 514-520.

Mhawej, M., Moog, C., Biafore, F., and Brunet-François, C., 2010. Control of the HIV infection and drug dosage. Biomed. Signal Proces. 5, 45- 52.

Miao, H., Hollenbaugh, J., Zand, M., Holden-Wiltse, J., Mosmann, T., Perelson, A., Wu, H., and Topham, D., 2010. Quantifying the early immune response and adaptive immune response kinetics in mice infected with influenza A virus. J. Virol. 84, 6687-6698.

Morrison, D., Roy, S., Rayner, C., Amer, A., Howard, D., Smith, J. R., and Evans, T. G., 2007. A randomized, crossover study to evaluate the pharmacokinetics of amantadine and oseltamivir administered alone and in combination. PLOS ONE 2, 1-6.

Moscona, A., 2005. Oseltamivir resistance -disabling our influenza defenses. N. Engl. J. Med. 353, 2636-2667.

Nitsch-Osuch, A., and LB, B., 2014. Influenza viruses resistant to neuraminidase inhibitors. Acta Biochimica Polonica. 61, 505-508.

Ogata, K., 2010. Modern control engineering. New Jersey: Prentice Hall.

Pawelek, K., Huynh, G., Quinlivan, M., Cullinane, A., Rong, L., and Perelson, A., 2012. Modeling within-host dynamics of influenza virus infection including immune responses. PLoS Comput. Biol. 8, e1002588.

Peaper, D., and Landry, M., 2014. Rapid diagnosis of influenza: State of the art. Clin. Lab. Med. 34, 365-385.

Potter, C., 2001. A history of influenza. J. Appl. Microbiol. 91, 572-579. 
Price, I., Mochan-Keef, E., Swigon, D., Ermentrout, G., Lukens, S., Toapanta, F., Ross, T., and Clermont, G., 2015. The inflammatory response to influenza A virus (H1N1): An experimental and mathematical study. J. Theor. Biol. 374, 83-93.

Rice, J., 2007. Mathematical statistics and data analysis. USA: Cengage Learning.

Rivadeneira, P., and Moog, C., 2012. Impulsive control of single-input nonlinear systems with application to HIV dynamics. Appl. Math. Comput. 218, 8462-8474.

Roberts, N., 2001. Treatment of influenza with neuraminidase inhibitors: virological implications. Philos. Trans. R. Soc. Lond. B Biol. Sci. 356, 1895-1897.

Saenz, R., Quinlivan, M., Elton, D., Macrae, S., Blunden, A., Mumford, J., Daly, J., Digard, P., Cullinane, A., Grenfell, B., McCauley, J., Wood, J., and Gog, J., 2010. Dynamics of influenza virus infection and pathology. J. Virol. 84, 3974-3983.

Schaller, S., Lippert, J., Schaupp, L., Pieber, T., Schuppert, A., and Eissing, T., 2016. Robust PBPK/PD based model predictive control of blood glucose. IEEE Trans. Biomed. Eng. 63, 1492-1504.

Swan, G., 1990. Role of optimal control theory in cancer chemotherapy. Math. Biosci. 101, $237-284$.

Tamiflu, 2009. Tamiflu (R) (oseltamivir phosphate) capsules and for oral suspension. package insert. foster city, California: Roche laboratories inc., gilead sciences inc. available online at:. http://www.fda.gov/downloads/Drugs/DrugSafety/DrugShortages/UCM183850.pdf.

Tchuenche, J. M., Khamis, S. A., Agusto, F. B., and Mpeshe, S. C., 2011. Optimal control and sensitivity analysis of an influenza model with treatment and vaccination. Acta Biotheoretica 59, 1-28.

Teixeira, R., and Malin, S., 2008. The next generation of artificial pancreas control algorithms. J. Diabetes Sci. Technol. 2, 105-112.

Toapanta, F., and Ross, T., 2009. Impaired immune responses in the lungs of aged mice following influenza infection. Respir. Res. 10, 112-131.

Wang, R., and Taubenberger, J., 2010. Methods for molecular surveillance of influenza. Expert Rev. Anti. Infect. Ther. 8, 517-527.

Ward, P., Small, I., Smith, J., Suter, P., and Dutkowski, R., 2005. Oseltamivir (Tamiflu) and its potential for use in the event of an influenza pandemic. J. Antimicrob. Chemother. 55: Suppl 1, i5-i21. 
Wattanagoon, Y., Stepniewska, K., Lindegårdh, N., Pukrittayakamee, S., Silachamroon, U., Piyaphanee, W., Singtoroj, T., Hanpithakpong, W., Davies, G., Tarning, J., Pongtavornpinyo, W., Fukuda, C., Singhasivanon, P., Day, N., and White, N., 2009. Pharmacokinetics of high-dose oseltamivir in healthy volunteers. Antimicrob. Agents Chemother. 53, 945-952.

World Health Organization, 2009. WHO guidelines for pharmacological management of pandemic (H1N1) 2009: Influenza and other influenza viruses. World Health Organization, Geneva.

Yang, T., 2001. Impulsive control theory. Berlin: Springer-Verlag.

Zhou, J., and Wen, C., 2008. Adaptive backstepping control of uncertain systems. Berlin: Springer-Verlag.

Zurakowski, R., and Teel, A., 2006. A model predictive control based scheduling method for HIV therapy. J. Theor. Biol. 238, $368-382$. 


\section{Figures}

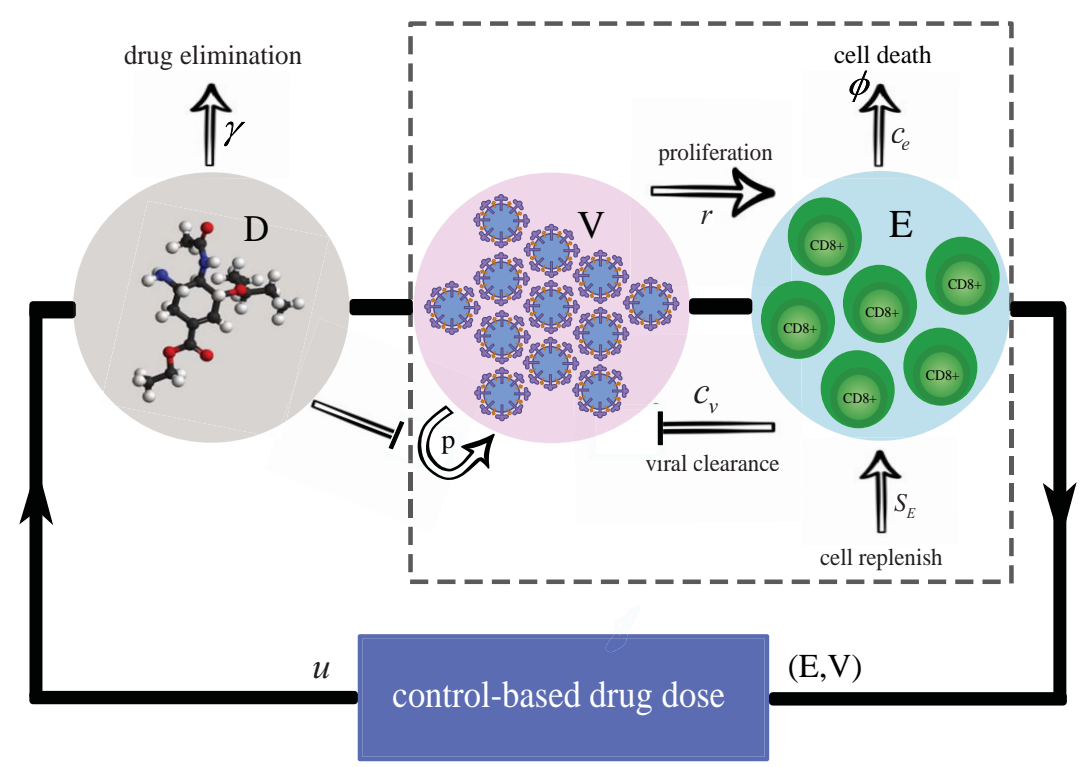

Figure 1. Scheme of adaptive feedback control loop. The closed-loop interaction between the drug, the virus and the immune response is shown. Gray dashed box: The virus replicates at rate $p$ (logistic growth, see Subsection 2.1) and induces more immune effector cells (collected here as CTLs) at rate $r$. CTLs suppress viral replication at rate $c_{v}$, die at rate $c_{e}$, and are replenished at rate $S_{E}$. The drug inhibits viral replication at rate $p$. The appropriate dose $u$ is calculated adaptively in the control-based drug dose unit from the measured virus concentration and the number of CTLs at each time of drug administration. 
a

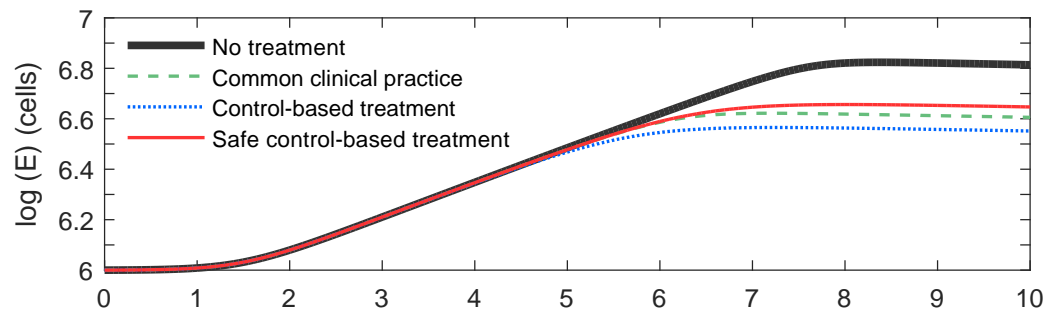

b

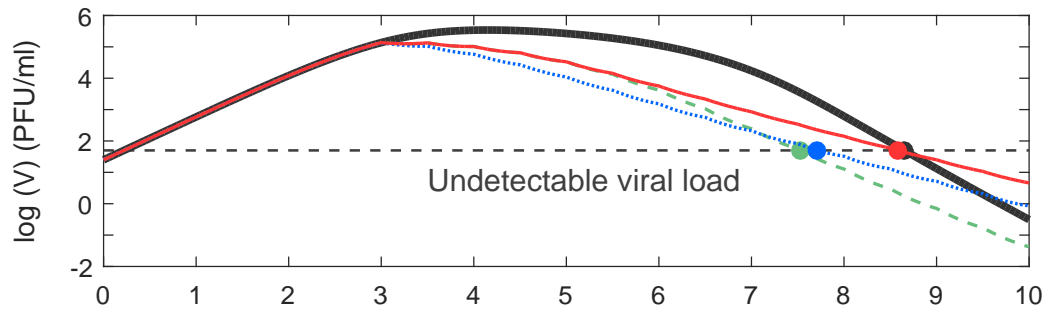

C

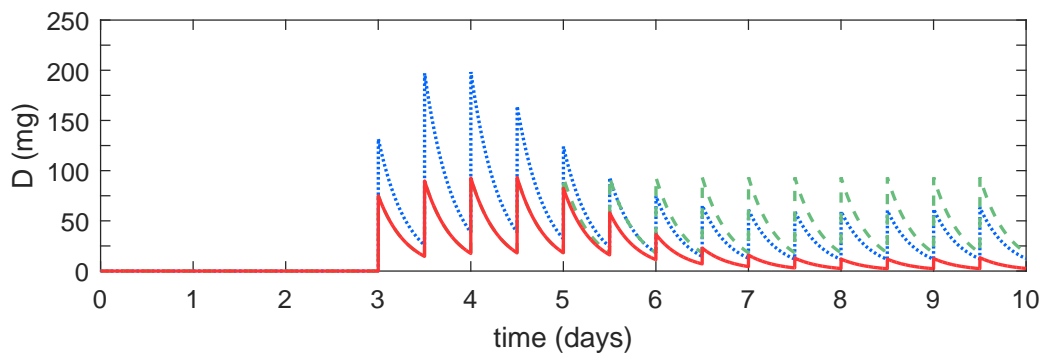

Figure 2. Low dose oseltamivir treatments started on day 3 after infection. a) Immune response, b) viral load and c) drug dynamics under the three different drug administration strategies for the low dose common clinical practice with $\Delta \mathrm{D}_{\text {fixed }}=75 \mathrm{mg}$ and imposing $\Delta \mathrm{D}_{\text {adaptive }} \leq 75 \mathrm{mg}$ in the safe control-based treatment. The viral infection is assumed to be cleared when $V \leq 50$ copies per $\mathrm{ml}$ are reached. Note that in the long term, the CTL count in the model returns to healthy conditions (not shown). Compared to the common clinical practice, the control-based treatment clears the infection equally fast, while keeping the number of CTLs lower. Under the safe control-based treatment, viral clearance is delayed by one day, but the drug dose is visibly reduced at later times of the infection. 
a

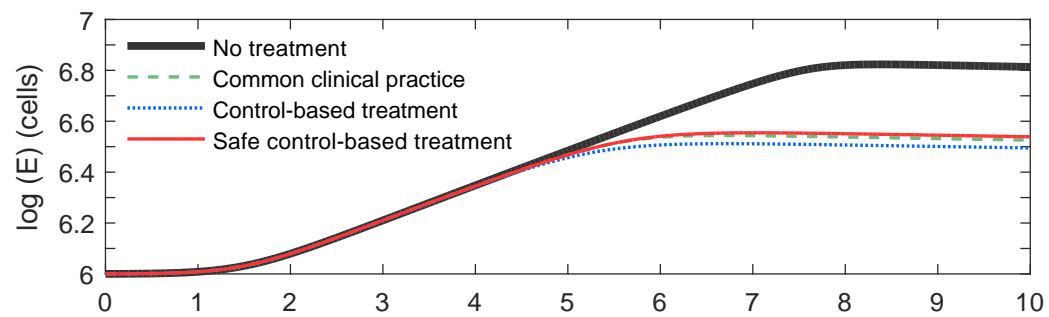

b

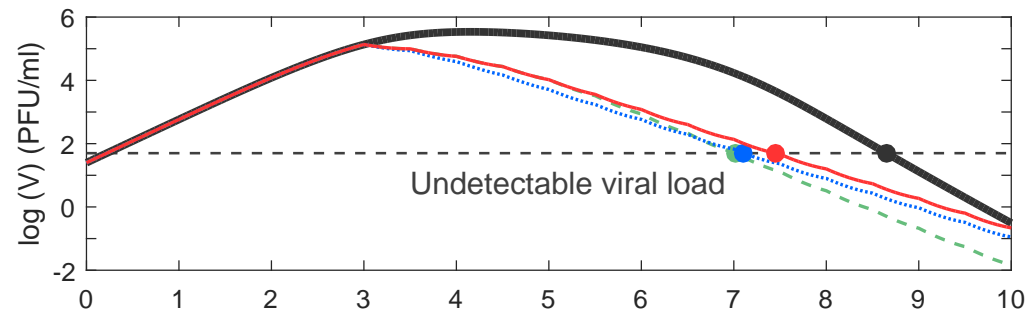

C

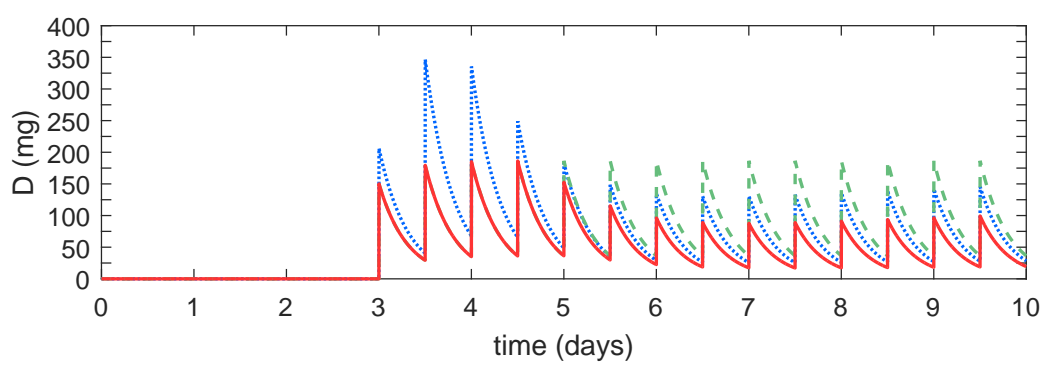

Figure 3. High dose oseltamivir treatments started on day 3 after infection. a) Immune response, b) viral load and c) drug dynamics under the three different drug administration strategies for the high dose common clinical practice with $\Delta \mathrm{D}_{\text {fixed }}=150 \mathrm{mg}$ and imposing $\Delta \mathrm{D}_{\text {adaptive }} \leq 150 \mathrm{mg}$ in the safe control-based treatment. The overall higher dose reduces differences in the CTLs level in all cases. The safe control-based treatment clears the infection equally fast compared to the other two treatment strategies with a lower amount of the drug. 
a

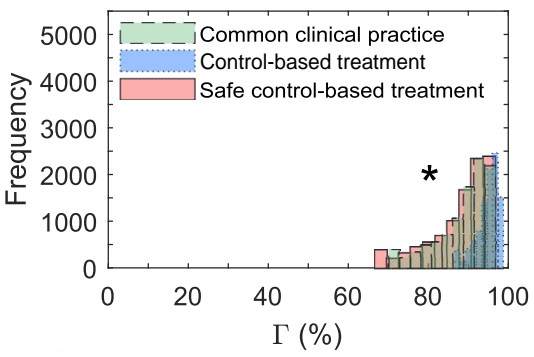

C

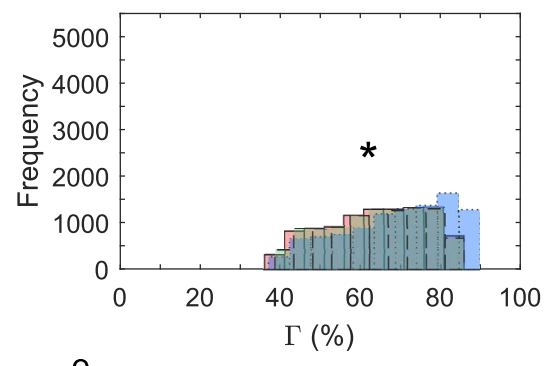

e

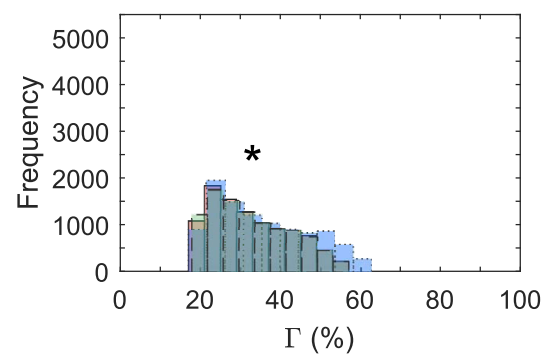

b

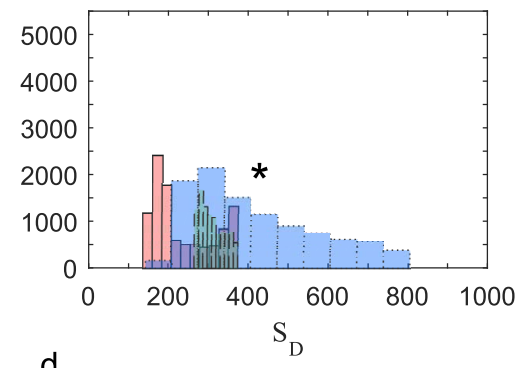

d

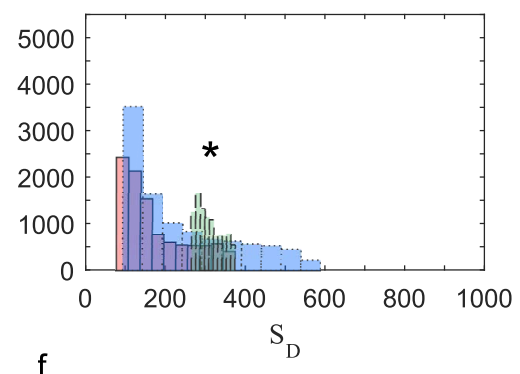

f

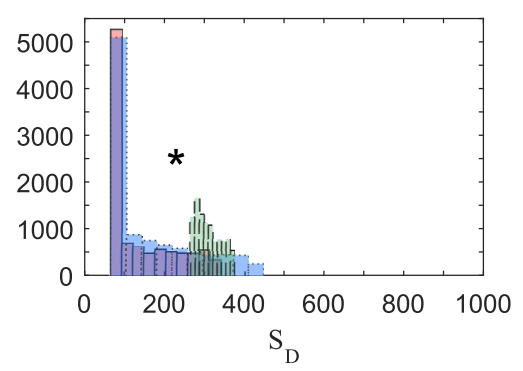

Figure 4. Monte Carlo simulations (with uniform distribution) of low dose treatments. Histograms of virological efficacy index $\Gamma$ and total administered drug (area under the drug curve $\mathrm{S}_{\mathrm{D}}$ ) of Monte Carlo analysis reflecting the diversity of infected hosts. The different treatment strategies are shown with the same color code and line style as before. Treatment was started on day 2,3 , or 4 in panels $(\mathrm{a}, \mathrm{b}),(\mathrm{c}, \mathrm{d})$, and $(\mathrm{e}, \mathrm{f})$, respectively. Simulations were performed with $\Delta \mathrm{D}_{\text {fixed }}=75 \mathrm{mg}$ for the low dose common clinical practice and with $\Delta \mathrm{D}_{\text {adaptive }} \leq 75 \mathrm{mg}$ for the safe control-based treatment. $(*)$ denotes the statistical significance between different treatments based on the one-way ANOVA test ( $p$-value $<\frac{0.05}{3}$ ). The control-based treatment in a,c,e and the safe control-based treatment in b,d,f show better performances compared to the common clinical practice. 
a

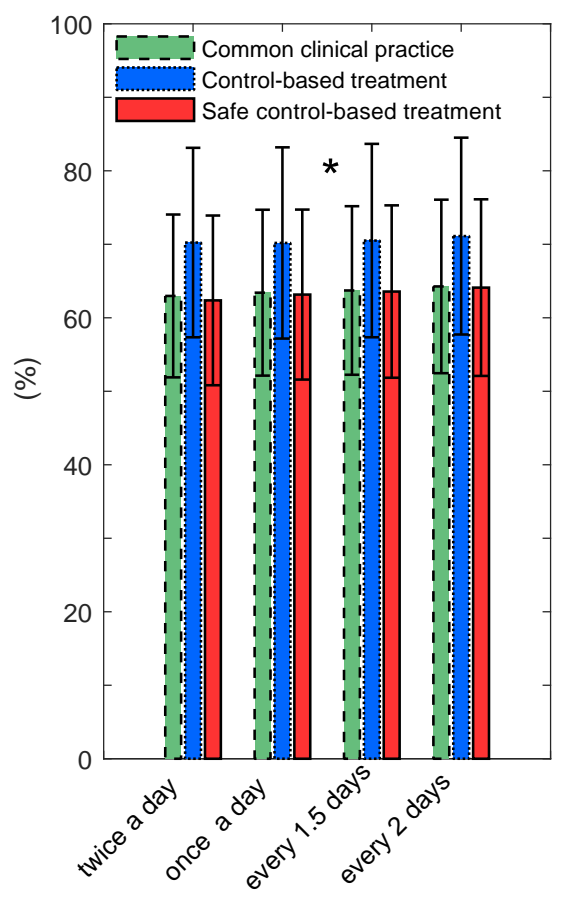

b

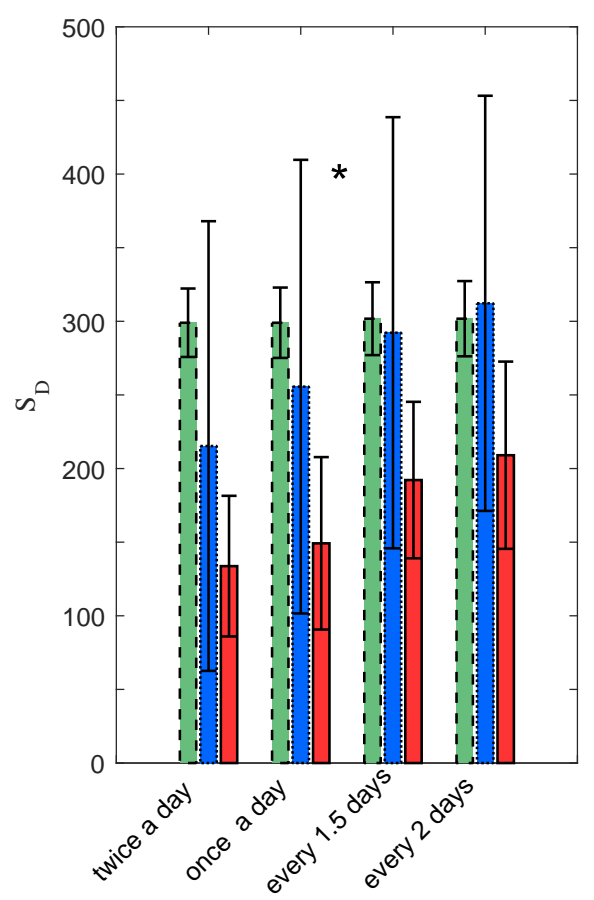

time intervals of drug dose update (days)

Figure 5. Low dose oseltamivir treatments (started on day 3 after infection) with different time intervals of drug dose update. Histograms and error bars of a) virological efficacy index $\Gamma$ and $b$ ) total administered drug (area under the drug curve $\mathrm{S}_{\mathrm{D}}$ ) for Monte Carlo simulations under the three different drug administration strategies for the low dose common clinical practice with $\Delta \mathrm{D}_{\text {fixed }}=75 \mathrm{mg}$ and imposing $\Delta \mathrm{D}_{\text {adaptive }} \leq 75 \mathrm{mg}$ in the safe control-based treatment. $(*)$ denotes the statistical significance between different treatments (with the same time interval of the drug update) based on the one-way ANOVA test (p-value $<\frac{0.05}{3}$ ). The adaptive strategies are robust against drug updating time intervals. The controlbased treatment and the safe control-based treatment show respectively higher and nearly equal $\Gamma$ compared to the common clinical practice for all updating intervals. Even though they choose higher doses as the updating interval increases, their good performances are preserved. 
a

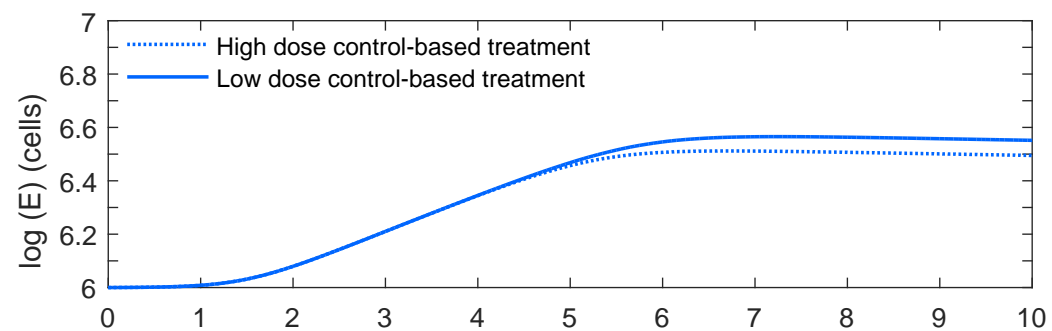

b

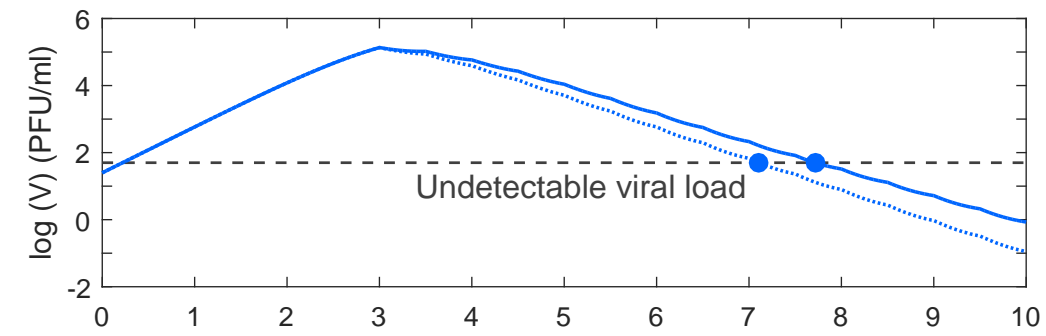

C

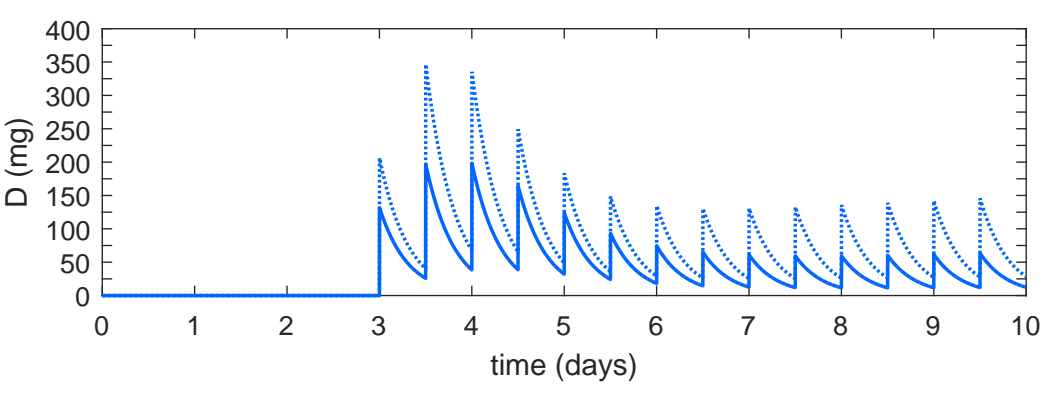

Figure S1. High dose versus low dose control-based treatment started on day 3 after infection. a) Immune response, b) viral load and c) drug dynamics. Adaptive dosing with a higher total amount of the drug results in faster viral clearance time and lower number of effector cells. 
a
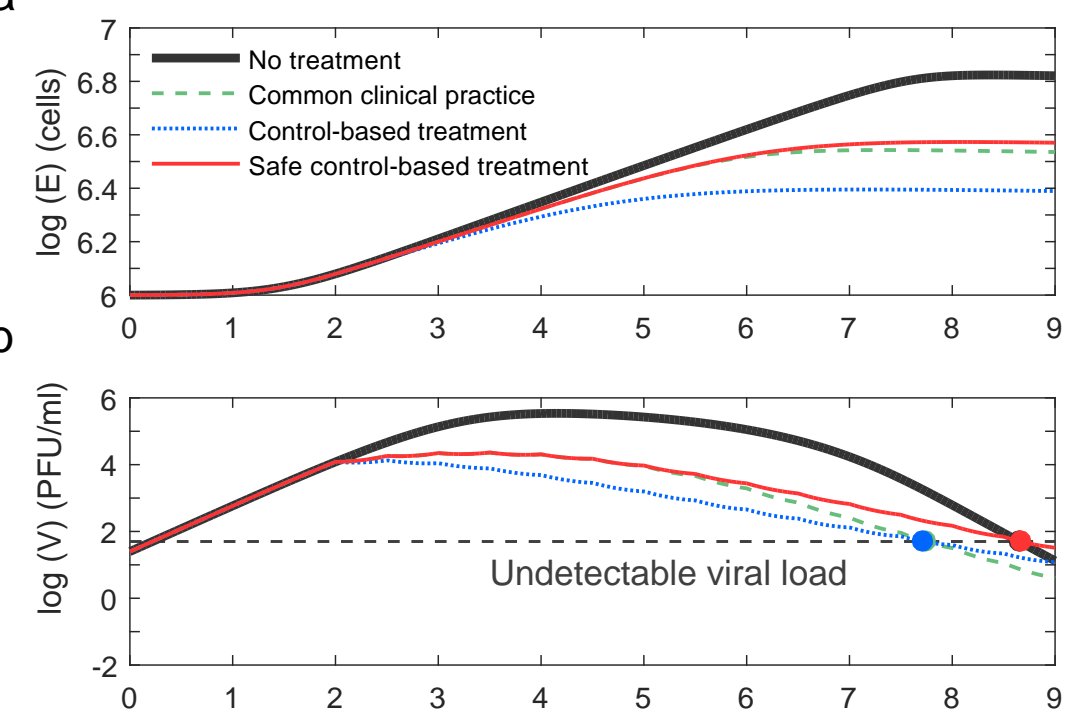

C

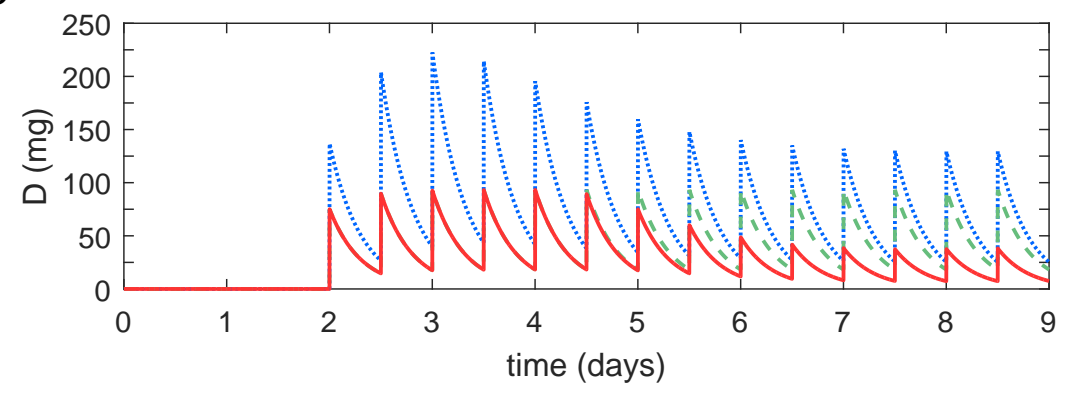

Figure S2. Low dose oseltamivir treatments started on day 2 after infection. a) Immune response, b) viral load and c) drug dynamics under the three different drug administration strategies for the low dose common clinical practice with $\Delta \mathrm{D}_{\text {fixed }}=75 \mathrm{mg}$ and imposing $\Delta \mathrm{D}_{\text {adaptive }} \leq 75 \mathrm{mg}$ in the safe control-based treatment. For earlier diagnosis, the adaptive strategies choose a higher amount of the drug to compensate for the weak immune response. 
a
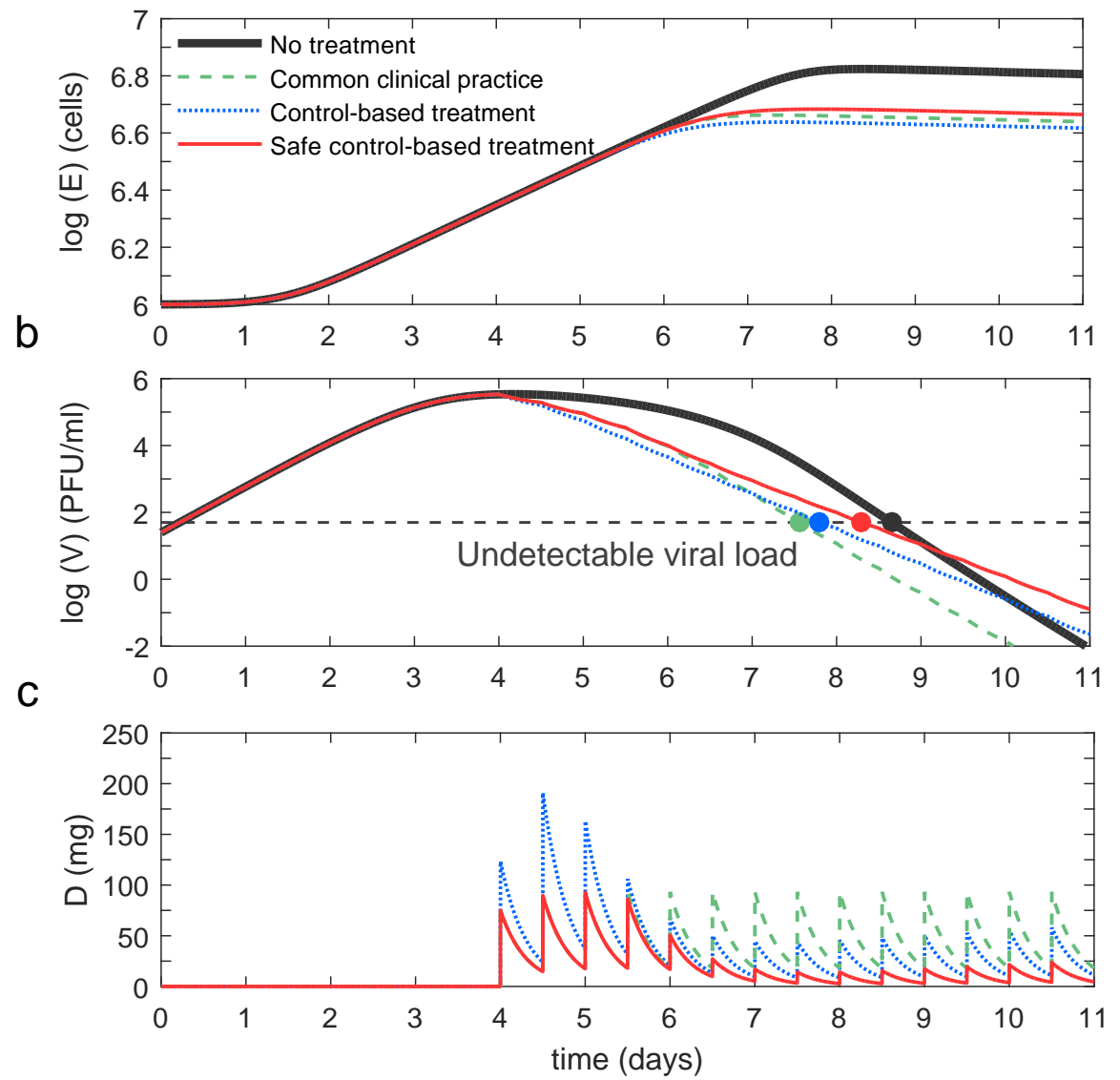

Figure S3. Low dose oseltamivir treatments started on day 4 after infection. a) Immune response, b) viral load and c) drug dynamics under the three different drug administration strategies for the low dose common clinical practice with $\Delta \mathrm{D}_{\text {fixed }}=75 \mathrm{mg}$ and imposing $\Delta \mathrm{D}_{\text {adaptive }} \leq 75 \mathrm{mg}$ in the safe control-based treatment. For late diagnosis, the adaptive strategies benefit from the stronger immune response and reduce the total administered drug. 

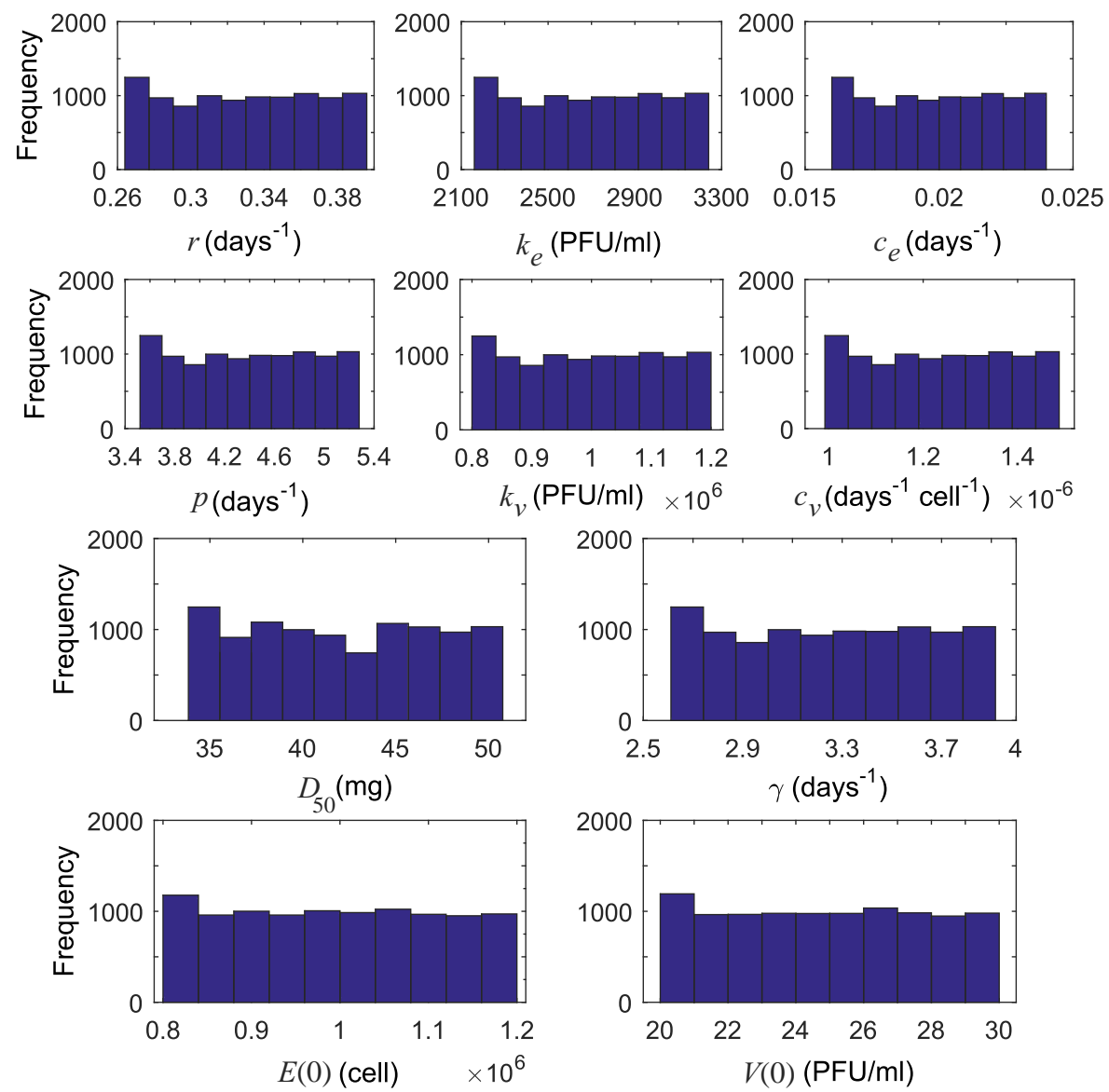

Figure S4. Variation of parameters and initial conditions taken from a uniform distribution. Histograms of random parameters/initial conditions in Monte Carlo analysis used to generate the results in figures 4 and S5. All parameters in the IAV-immune model (1)-(4) and the initial states $V(0)$ and $E(0)$ are selected uniformly from the interval $[\bar{m}-20 \% \bar{m}, \bar{m}+20 \% \bar{m}]$, where $\bar{m}$ is the median value of $m$ with $m \in\left\{r, k_{e}, c_{e}, p, k_{v}, c_{v}, D_{50}, \gamma, V(0), E(0)\right\}$. 

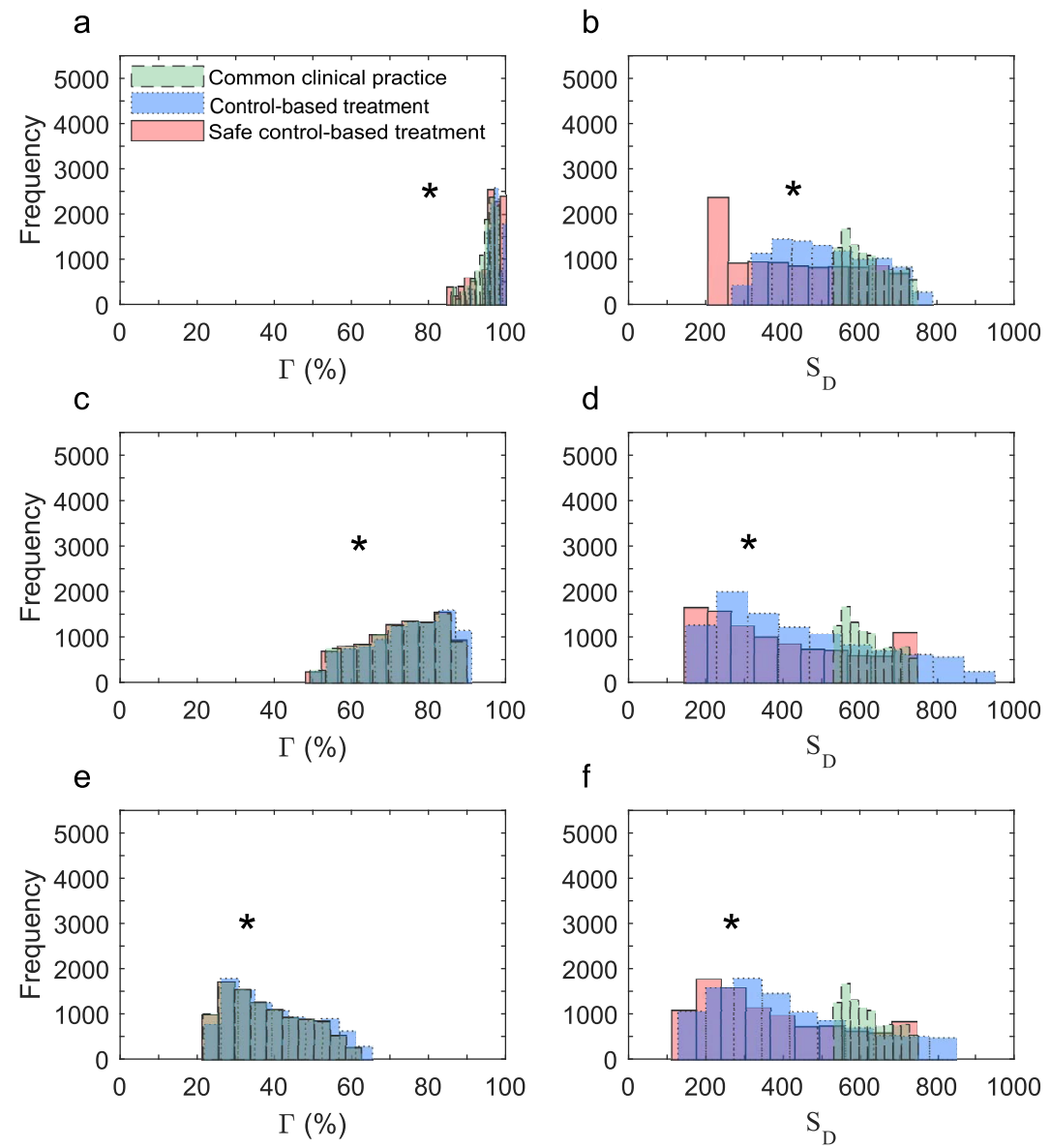

Figure S5. Monte Carlo simulations (with uniform distribution) of high dose treatments. Histograms of virological efficacy index $\Gamma$ and total administered drug (area under the drug curve $\mathrm{S}_{\mathrm{D}}$ ) of Monte Carlo analysis reflecting the diversity of infected hosts. The different treatment strategies are shown with the same color code and line style as before. Treatment was started on day 2,3 , or 4 in panels $(\mathrm{a}, \mathrm{b}),(\mathrm{c}, \mathrm{d})$, and $(\mathrm{e}, \mathrm{f})$, respectively. Simulations were performed with $\Delta \mathrm{D}_{\text {fixed }}=150 \mathrm{mg}$ for the low dose common clinical practice and with $\Delta \mathrm{D}_{\text {adaptive }} \leq 150 \mathrm{mg}$ for the safe control-based treatment. $(*)$ denotes the statistical significance between different treatments based on the one-way ANOVA test $\left(\mathrm{p}\right.$-value $\left.<\frac{0.05}{3}\right)$. The control-based treatment in a,c,e and the safe control-based treatment in b,d,f show better performances compared to the common clinical practice. 

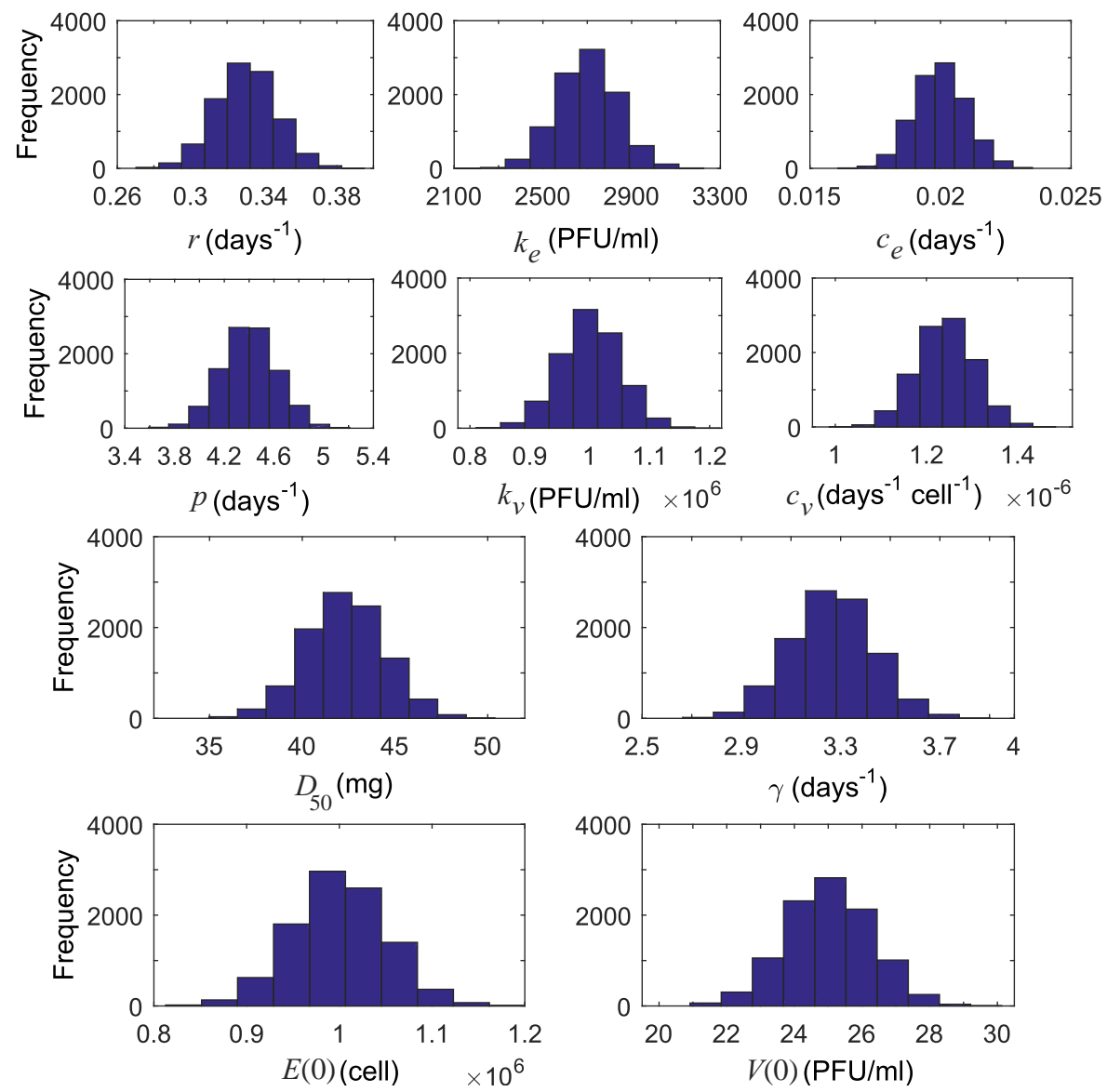

Figure S6. Variation of parameters and initial conditions taken from a normal distribution. Histograms of random parameters/initial conditions in Monte Carlo analysis used to generate the results in figures S7 and S8. All parameters in the IAV-immune model (1)-(4) and the initial states $V(0)$ and $E(0)$ are selected from a normal distribution with median $\bar{m}$ and standard deviation $0.05 \bar{m}$. 
a

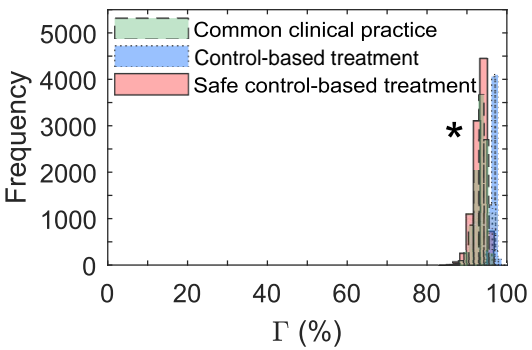

C
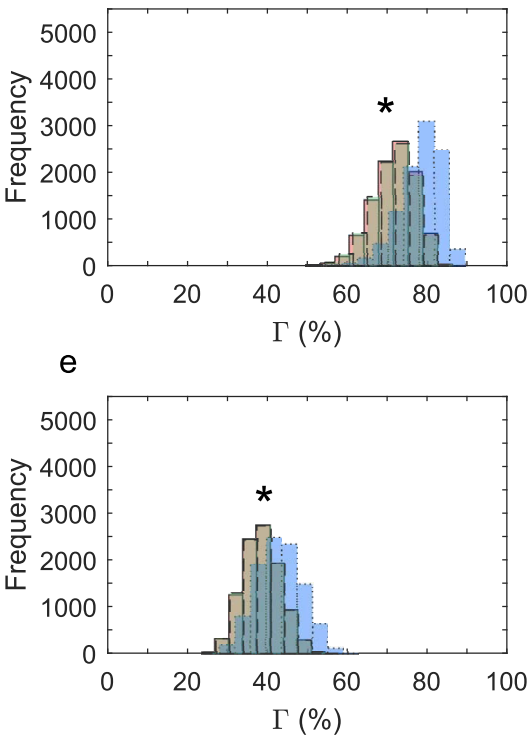

b

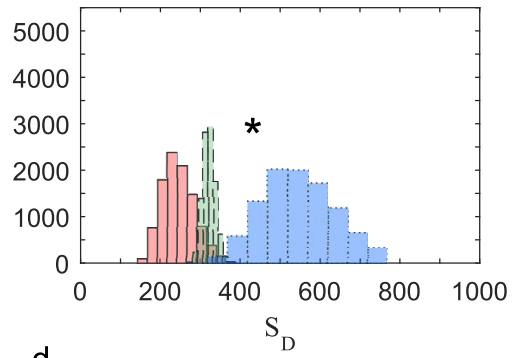

d

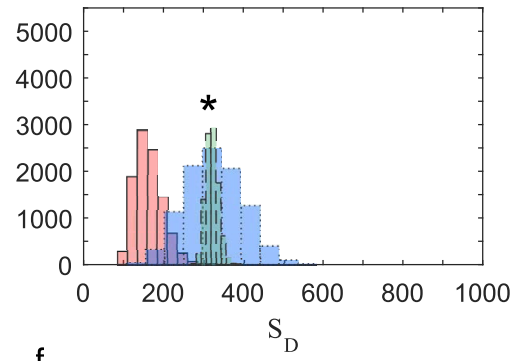

f

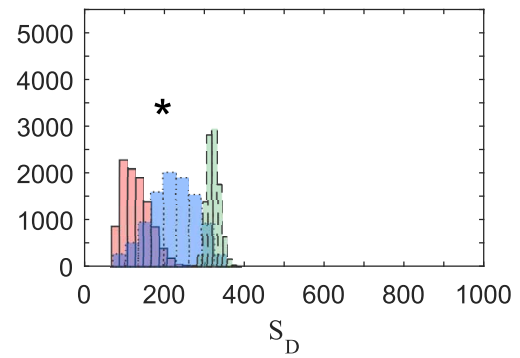

Figure S7. Monte Carlo simulations (with normal distribution) of low dose treatments. Histograms of virological efficacy index $\Gamma$ and total administered drug (area under the drug curve $\mathrm{S}_{\mathrm{D}}$ ) of Monte Carlo analysis reflecting the diversity of infected hosts. The different treatment strategies are shown with the same color code and line style as before. Treatment was started on day 2,3 , or 4 in panels $(\mathrm{a}, \mathrm{b}),(\mathrm{c}, \mathrm{d})$, and $(\mathrm{e}, \mathrm{f})$, respectively. Simulations were performed with $\Delta \mathrm{D}_{\text {fixed }}=75 \mathrm{mg}$ for the low dose common clinical practice and with $\Delta \mathrm{D}_{\text {adaptive }} \leq 75 \mathrm{mg}$ for the safe control-based treatment. $(*)$ denotes the statistical significance between different treatments based on the one-way ANOVA test ( $\mathrm{p}$-value $<\frac{0.05}{3}$ ). The control-based treatment in a,c,e and the safe control-based treatment in b,d,f show better performances compared to the common clinical practice. Similar to the results with the uniform distribution, the improved performance of the adaptive strategies is preserved. 
a

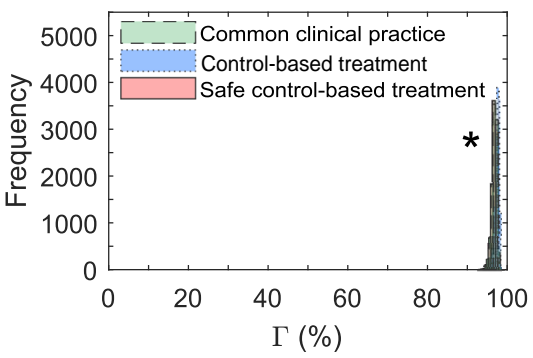

C

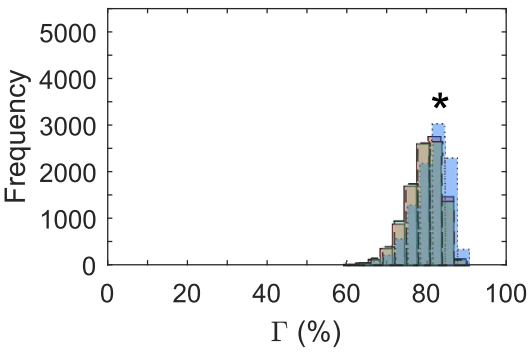

e

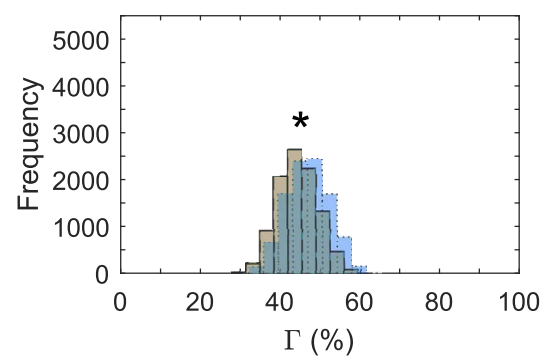

b

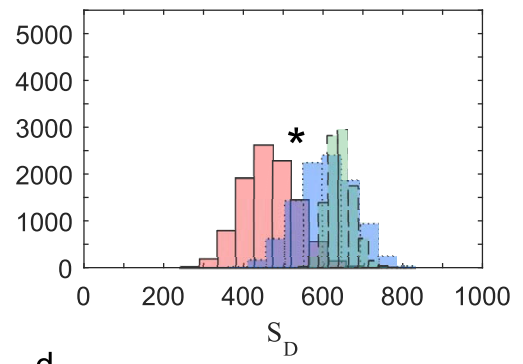

d
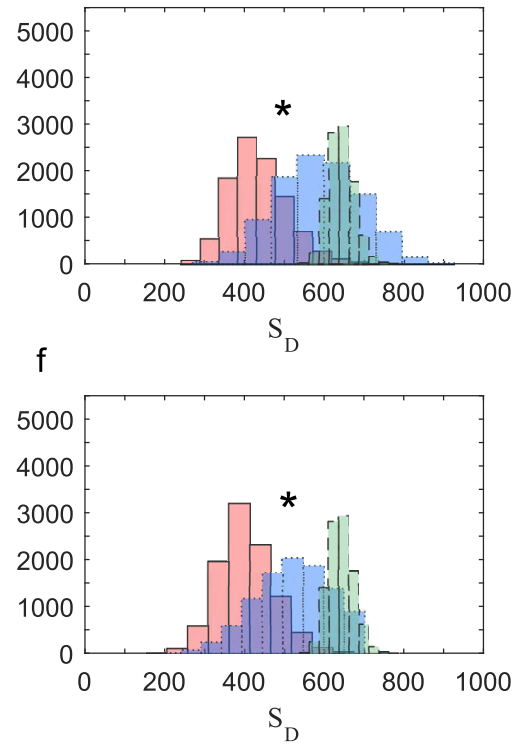

Figure S8. Monte Carlo simulations (with normal distribution) of high dose treatments. Histograms of virological efficacy index $\Gamma$ and total administered drug (area under the drug curve $\mathrm{S}_{\mathrm{D}}$ ) of Monte Carlo analysis reflecting the diversity of infected hosts. The different treatment strategies are shown with the same color code and line style as before. Treatment was started on day 2,3 , or 4 in panels (a,b), (c,d), and (e,f), respectively. Simulations were performed with $\Delta \mathrm{D}_{\text {fixed }}=150 \mathrm{mg}$ for the low dose common clinical practice and with $\Delta \mathrm{D}_{\text {adaptive }} \leq 150 \mathrm{mg}$ for the safe control-based treatment. (*) denotes the statistical significance between different treatments based on the one-way ANOVA test (p-value $<\frac{0.05}{3}$ ). The control-based treatment in a,c,e and the safe control-based treatment in b,d,f show better performances compared to the common clinical practice. Similar to the results with the uniform distribution, the improved performance of the adaptive strategies is preserved. 
a

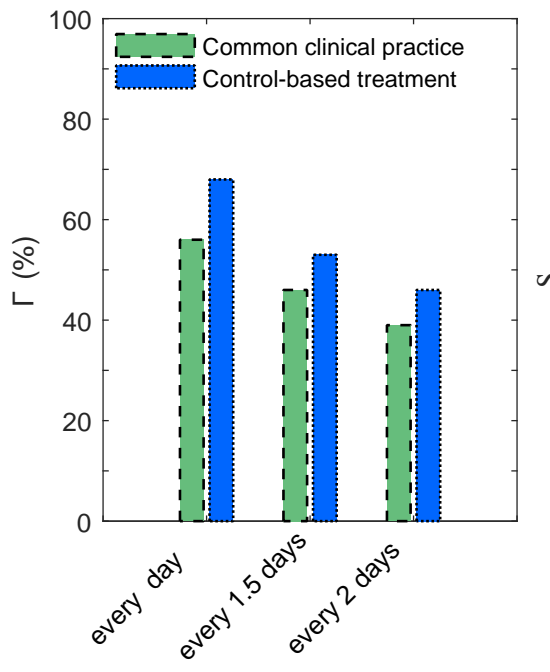

b

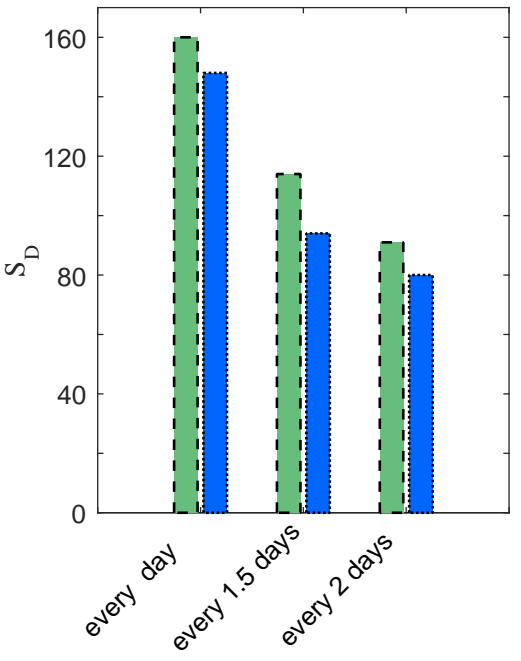

time intervals of drug administration (days)

Figure S9. Low dose oseltamivir treatments (started on day 3 after infection) with different time intervals of drug administration. Histograms of a) virological efficacy index $\Gamma$ and $b$ ) total administered drug (area under the drug curve $\mathrm{S}_{\mathrm{D}}$ ) for the common clinical practice and the control-based treatment with the low dose. Unlike in Fig. 5, here, we assume the same time interval for both drug administration and drug dose update. For each time interval, the control-based treatment highlights its performance by showing the higher virological efficacy $\Gamma$ while using a lower amount of the drug. 
a

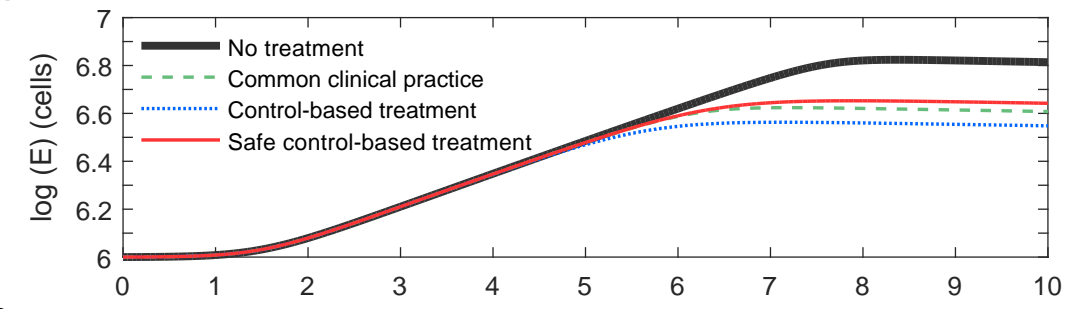

b

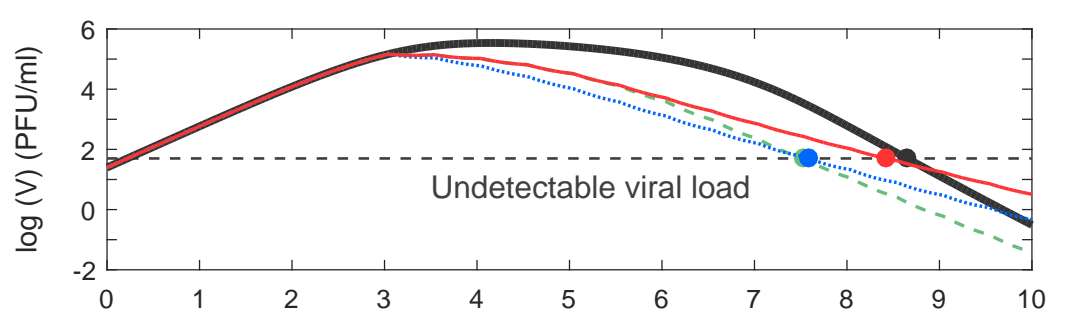

C

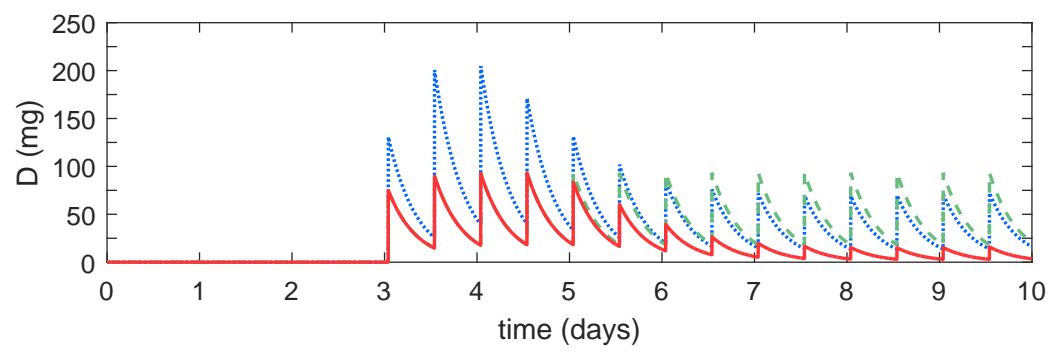

Figure S10. Low dose oseltamivir treatments (started on day 3 after infection) considering one hour delay in the oseltamivir absorption. a) Immune response, b) viral load and c) drug dynamics under the three different drug administration strategies for the low dose common clinical practice with $\Delta \mathrm{D}_{\text {fixed }}=75 \mathrm{mg}$ and imposing $\Delta \mathrm{D}_{\text {adaptive }} \leq 75$ $\mathrm{mg}$ in the safe control-based treatment. We assume that each drug dose becomes effective with one hour delay from the time of administration. Compared to the corresponding results without delay (Fig. 2), the adaptive treatment strategies preserve their performance even in the presence of the drug absorption phase. The only difference is that here, the adaptive treatment strategies select higher doses to compensate for the considered delay. This in turn leads to the faster viral clearance. 
a

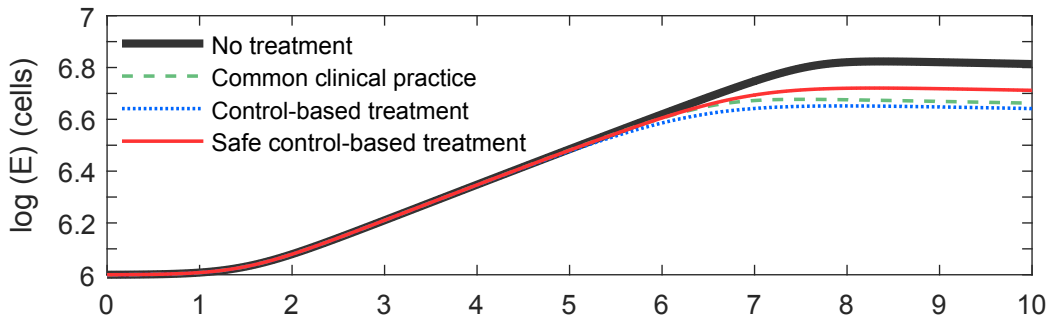

b

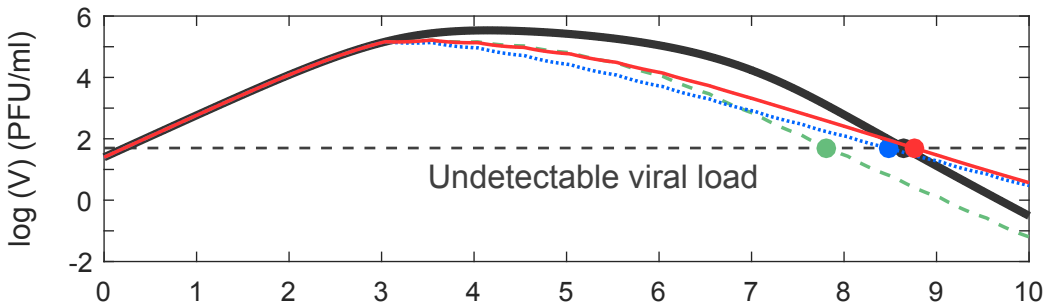

C

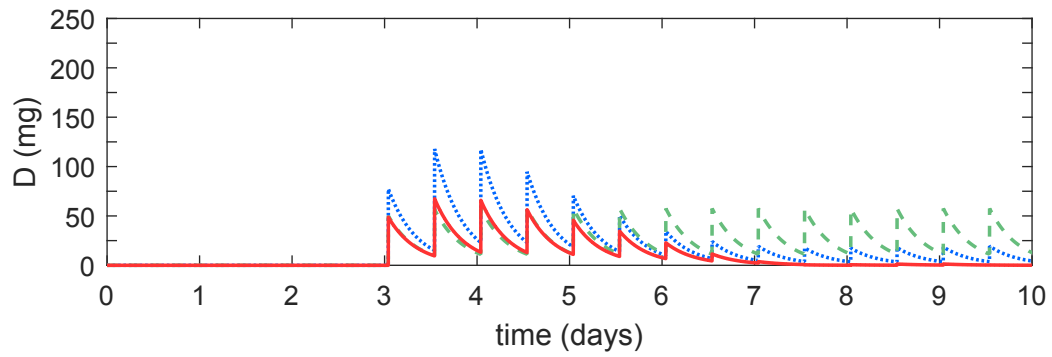

Figure S11. Low dose oseltamivir treatments (started on day 3 after infection) considering drug elimination during the absorption time. a) Immune response, b) viral load and c) drug dynamics under the three different drug administration strategies. We assume that $2 / 3$ of each drug dose becomes effective with one hour delay from the time of administration and the remaining drug is cleared from the bloodstream. Compared to the corresponding results without considering the drug elimination (Fig. S10), the drug doses decrease and this leads to a slower viral load clearance. The safe control-based treatment selects small (non-zero) doses from day 7 but it can still achieve the same virological efficacy as in the common clinical practice (see Table S3). 